\title{
Effects of Surface Roughness on Airframe Noise
}

\author{
Yu Liu*, Ann P. Dowling ${ }^{\dagger}$ and Ho-Chul Shin ${ }^{\ddagger}$ \\ University of Cambridge, Cambridge, CB2 1PZ, Great Britain
}

\begin{abstract}
The generation of sound by turbulent boundary layer flow at low Mach number over a rough wall is investigated by applying the theoretical model which describes the scattering of the turbulence near field into sound by roughness elements. Attention is focused on a numerical method to approximately quantify the absolute level of the roughness noise radiated to far field. Empirical models for the source statistics are obtained by scaling smooth-wall data by the increased skin friction velocity and boundary layer thickness for a rough surface. Numerical integration is performed to determine the roughness noise, and it reproduces the spectral characteristics of the available empirical formula and experimental data. Experiments are conducted to measure the radiated sound from two rough plates in an open jet by four $1 / 2$ " free-field condenser microphones. The measured noise spectra of the rough plates are above that of a smooth plate in $1-2.5 \mathrm{kHz}$ frequency and exhibits encouraging agreement with the predicted spectra. Also, a phased microphone array is utilized to localize the sound source, and it confirms that the rough plates have higher acoustic source strengthes in this frequency range. A parametric study illustrates that the roughness height and roughness density significantly affect the far-field radiated roughness noise with the roughness height having the dominant effect. The estimates of the roughness noise for a Boeing 757 sized aircraft wing with idealized levels of surface roughness show that in high frequency region the sound radiated from surface roughness may exceed that from the trailing edge, and higher overall sound pressure levels for the roughness noise are also observed.
\end{abstract}

\section{Nomenclature}

$\boldsymbol{v} \quad$ Perturbation velocity

$\boldsymbol{x} \quad$ Observer position

$\boldsymbol{Y} \quad$ Velocity potential of an ideal incompressible flow over the rough wall

$\boldsymbol{y} \quad$ Source position

$\tilde{\boldsymbol{n}} \quad$ Unit normal vector

$A \quad$ Area of the rough wall

$A_{j} \quad$ The $j$ th equal subarea of the rough region $=A / N_{A}$

$B \quad$ Empirical constant for the mean velocity profile on a rough wall $=8.5$

$c \quad$ Speed of sound in free field

$C_{A} \quad$ A-weighting factor

$C_{F} \quad$ Overall skin friction coefficient $=\frac{1}{L} \int_{0}^{L} c_{f} d x_{1}$

$c_{f} \quad$ Local skin friction coefficient $=\tau_{w} / \frac{1}{2} \rho_{0} U^{2}$

$c_{f 0} \quad$ Local skin friction coefficient at a distance $x_{l e}$ from the leading edge

$D \quad$ Directivity function

$f \quad$ Frequency

$G \quad$ Green function

$I_{1}-I_{3} \quad$ Integrals with respect to the polar coordinates $\kappa$ and $\alpha$ of the wavenumber plane

$J_{1} \quad$ Bessel function of order unity

*Ph.D. Research Student, Department of Engineering, Student Member AIAA.

$\dagger$ Professor, Department of Engineering, Member AIAA.

${ }^{\ddagger}$ Research Associate, Department of Engineering, Member AIAA. 
$k_{0} \quad$ Acoustic wavenumber $=\omega / c$

$k_{s} \quad$ Equivalent roughness height

$L \quad$ Length of the flat plate

$L_{c} \quad$ Equivalent chord of an aircraft wing

$L_{s} \quad$ Equivalent span of an aircraft wing

$M \quad$ Mach number $=U / c$

$N \quad$ Average number of roughness bosses per unit area

$n \quad$ Empirical coefficient in $\Phi_{R}$, see Eq. (37)

$N_{A} \quad$ Number of subareas

$P \quad$ Rough-wall wavenumber-frequency spectrum

$p \quad$ pressure fluctuation

$P_{R} \quad$ Acoustic frequency spectrum of far-field radiated roughness noise

$P_{s} \quad$ Smooth-wall wavenumber-frequency spectrum

$p_{s} \quad$ Hypothetical smooth-wall pressure fluctuation

$Q \quad$ Turbulence Reynolds stress source

$R \quad$ Roughness height

$R e_{\tau} \quad$ Roughness Reynolds number $=k_{s} u_{\tau} / \nu$

$R e_{L} \quad$ Reynolds number based on the whole plate $=U L / \nu$

$R e_{l e} \quad$ Reynolds number based on the distance between the rough region and leading edge $=U x_{l e} / \nu$

$S \quad$ Cross-spectral density of the turbulence Reynolds stress

$S h^{*} \quad$ Strouhal number based on the displacement boundary layer thickness $=\omega \delta^{*} / U$

$t$ time

$U \quad$ Free stream velocity

$u_{\tau} \quad$ Friction velocity

$U_{c} \quad$ Eddy convection velocity

$v \quad x_{2}$-component of the perturbation velocity

$w \quad$ Wake function

$x_{0} \quad$ Hypothetical extension of the rough region

$x_{l e} \quad$ Distance between the rough region and the leading edge

\section{Subscripts}

$\eta \quad$ Summation variable over $x_{1}$ and $x_{3}$ in plane directions

$i, j \quad$ General summation variable

$m \quad$ Summation variable over the roughness bosses

\section{Symbols}

$\alpha \quad$ Polar angle of the wavenumber plane

$\beta \quad$ Empirical coefficient in $\Phi_{R}$, see Eq. (37)

$\boldsymbol{\kappa} \quad$ Wavenumber vector $=\left(\kappa_{1}, 0, \kappa_{3}\right)$

$\boldsymbol{\Delta} \quad$ Space separation vector

$\delta \quad$ Local boundary layer thickness

$\delta(\boldsymbol{x}) \quad$ Dirac function

$\delta^{*} \quad$ Displacement boundary layer thickness

$\delta_{0} \quad$ Boundary layer thickness at the front edge of the rough region

$\gamma \quad=\left(k_{0}^{2}-\kappa^{2}\right)^{\frac{1}{2}}$

$\kappa \quad$ Amplitude of the wavenumber $\kappa$

$\kappa_{0} \quad$ Kármán constant $\approx 0.41$

$\lambda \quad$ Acoustic wavelength $=2 \pi c / \omega$

$\mu \quad$ Roughness density factor $=1 /\left(1+\frac{1}{4} \sigma\right)$

$\nu \quad$ Kinematic viscosity

$\Omega \quad$ Dimensionless frequency $=\omega R / u_{\tau}$

$\omega \quad$ Angular frequency

$\Phi \quad$ Point pressure frequency spectrum

$\tilde{\Phi}_{p} \quad$ Empirical model for the smooth-wall wavenumber-frequency spectrum

$\Phi_{R} \quad$ Empirical model for the rough-wall acoustic frequency spectrum

$\Pi \quad$ Rough-wall acoustic power spectrum 


$\begin{array}{ll}\Pi_{0} & \text { Wake strength } \\ \Psi & \text { Term related to the average number of bosses per unit area } \\ \rho_{0} & \text { Mean fluid density in free field } \\ \sigma & \text { Roughness density }=N \pi R^{2} \\ \sigma_{p} & \text { Peak value of } \sigma \text { where the OASPL achieves the maximum } \\ \sigma_{\max } & \text { Maximum value of } \sigma \text { for a hexagonal close packing } \sim 0.91 \\ \sigma_{s q} & \text { The value of } \sigma \text { for a square close packing } \sim 0.78 \\ \tau & \text { Time delay } \\ \tau_{0} & \text { Roughness parameter } \\ \tau_{w} & \text { Mean wall shear stress }=\rho_{0} u_{\tau}^{2} \\ \theta, \phi & \text { Directivity angles } \\ \xi & \text { Definition of the rough surface } \\ - & \text { Mean value } \\ \hat{\langle} & \text { Fourier transform } \\ \langle\rangle & \text { Ensemble average } \\ * & \text { Normalized value } \\ * & \text { Complex conjugate }\end{array}$

M1-M4 1/2" free-field condenser microphones

OASPL Overall Sound Pressure Level, dBA

SPL Sound Pressure Level, dB

TE Trailing Edge

\section{Introduction}

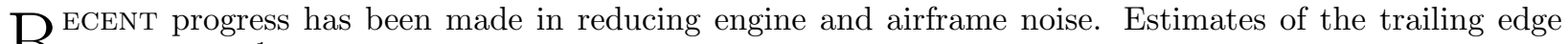
Rnoise are low ${ }^{1}$ and this means increasing interests in other features that may contribute to the noise of a "clean" configuration. Panel vibration may give noticeable noise levels. ${ }^{2}$ In this paper we look at another mechanism whereby energy in the turbulent boundary layer may scatter into radiated sound due to the presence of small surface roughness. This might arise from discontinuities (rivets, ribs, joints, etc.), environmental contamination, paint finishes or imprecise machining during manufacture. Relatively small degrees of surface roughness can produce substantial increases in drag compared to that predicted for the corresponding, perfectly smooth boundary. ${ }^{3}$ Similarly large increases will therefore be expected to occur in the strength of the boundary-layer generated noise.

Howe ${ }^{4}$ has previously presented a theoretical model on the generation of sound by turbulent boundary layer flow over a rough wall, and speculated that the surface roughness generated noise would be a substantial fraction of the airframe noise of an airplane flying in the "clean" configuration. In this theory, the rough surface is simply modeled by randomly distributing rigid, hemispherical bosses over an otherwise smooth plane. It is assumed that the turbulent boundary layer roughness noise is produced primarily by the diffraction of the turbulent hydrodynamic near field (pseudo-sound) by the roughness bosses. The analysis is according to the classical, ideal fluid diffraction theory so that the viscous stresses on the wall can be disregarded. This approximation is likely to be adequate for the surface roughness Reynolds number $R e_{\tau}=k_{s} u_{\tau} / \nu>10$, where $\nu$ is the kinematic viscosity, $u_{\tau}$ the friction velocity, and $k_{s}$ the equivalent roughness height determined by fitting a conventional logarithmic curve to the mean boundary-layer velocity profile. ${ }^{3}$ In this case the roughness bosses protrude beyond the viscous sublayer, and this is consistent with the usual criteria (namely $\left.k_{s} u_{\tau} / \nu>5\right)$ for a surface to be hydraulically rough.

Howe ${ }^{5}$ then extended the inviscid diffraction theory in Ref. 4 to ascertain the possible influence of viscous wall stresses on the diffraction mechanism. In these circumstances the roughness elements are assumed to be sufficiently small and contained entirely within the viscous sublayer (i.e. $k_{s} u_{\tau} / \nu<5$ ) that the viscous "no-slip" condition at the wall may be applied iteratively on the mean plane of the wall. By this means it is concluded that over the whole frequency range in which roughness noise is expected to be significant, viscous effects increase the levels of the radiated sound by 2 or $3 \mathrm{~dB}$ at the most.

An important limitation of the above theoretical approaches ${ }^{4,5}$ lies in the fact that they are unable to account for local effects such as the formation of wakes (vortex shedding) by the roughness elements, which 
creates new noise sources during the interaction of the turbulence with the wall. It is therefore anticipated that the diffraction theory would be adequate only for surfaces whose roughness elements do not penetrate beyond the buffer zone into the fully developed turbulent region of the flow. In this case the acoustic frequency spectrum $P_{R}(\boldsymbol{x}, \omega)$ of the far-field radiated roughness noise can be expressed as an infinite integral in terms of the smooth-wall wavenumber-frequency spectrum $P_{s}(\boldsymbol{\kappa}, \omega)$ of the hydrodynamic pressure fluctuations on a control surface located at a distance $R$ from the mean wall plane ${ }^{\mathrm{a}}$.

There have been a number of empirical models already for the smooth-wall wavenumber-frequency spectrum. Graham ${ }^{6}$ discussed the different models presented by Corcos, ${ }^{7}$ Efimtsov ${ }^{8}$ Smol'yakov and Tkachenko, ${ }^{9}$ Ffowcs Williams,${ }^{10}$ and Chase. ${ }^{11,12}$ These models express $P_{s}(\boldsymbol{\kappa}, \omega)$ in terms of the friction velocity $u_{\tau}$, boundary layer thickness $\delta$, and eddy convection velocity $U_{c}$, etc. For moderately rough surfaces, we make the same assumption as Howe ${ }^{4}$ that the principal differences in the features of the wall pressure spectrum from those of the wall pressure spectrum on a smooth wall are accounted for by the differences in $u_{\tau}$ and $\delta$. This is expected to be a good approximation especially in the vicinity of the convective peak. ${ }^{11}$ Therefore in this approach the rough-wall wavenumber-frequency spectrum is approximated by a smooth-wall empirical formula provided that $u_{\tau}$ and $\delta$ are increased to compensate for the increased surface drag and turbulence production. ${ }^{3}$

The integral of $P_{R}(\boldsymbol{x}, \omega)$ was conventionally evaluated by means of asymptotic approximation ${ }^{4,13}$ based on the wall pressure spectrum being sharply peaked in the vicinity of the convective ridge. An empirical formula for $P_{R}(\boldsymbol{x}, \omega)$ was also proposed by Howe ${ }^{13}$ and the values of adjustable coefficients were partially estimated by the experimental data of $\mathrm{Hersh}^{14}$ on the sound radiation by sand-roughened pipes of various grit sizes. However, not all the coefficient values have been determined yet due to insufficient experimental data; hence the absolute level of $P_{R}(\boldsymbol{x}, \omega)$ has been unable to be determined so far.

Therefore, the primary objective of this paper is to find a reliable scheme to approximately predict the far-field radiated roughness noise both in the spectral shape and in the absolute level, and then apply it to the investigation of surface roughness effects on airframe noise. In this paper a generally more accurate method was adopted to evaluate $P_{R}(\boldsymbol{x}, \omega)$ by direct numerical integration in the wavenumber space. Furthermore, experiments are presented to validate this numerical scheme. Hence the acoustic measurements with $1 / 2$ " free-field condenser microphones and a phased microphone array were conducted to enable the comparison between the measured and predicted roughness noise spectra. Following the experimental validation, the numerical scheme was used to estimate the far-field roughness noise for an aircraft wing with different levels of surface roughness, and the relative levels of the roughness noise and trailing edge noise are discussed.

\section{Diffraction Theory of Turbulent Boundary Layer Roughness Noise}

\section{A. Formulation of the Diffraction Problem}

The diffraction theory of turbulent boundary layer roughness noise is based on the theoretical model of Howe, ${ }^{4}$ and the major derivations will be presented here for the sake of completeness. The general idea of the diffraction of roughness generated sound is introduced by considering turbulent boundary layer flow over a rough, rigid wall defined by the surface $x_{2}=\xi\left(x_{\eta}\right)$, where Greek suffix $\eta$ varies over the 1-direction and 3 -direction parallel to the mean plane of the wall. The rough wall is formed by a random distribution $(N$ per unit area) of hemispherical bosses of radius $R$ over the plane $x_{2}=0$ (see figure 1), and the fluid occupies the region $x_{2}>\xi\left(x_{\eta}\right)$. The mean flow is in the positive $x_{1}$-direction, and is of uniform mean density $\rho_{0}$ and sufficiently low Mach number that pressure fluctuations $p$ are related to turbulent fluctuations in the Reynolds stress $\rho_{0} v_{i} v_{j}$ by Lighthill's equation in the reduced form:

$$
\left(\frac{1}{c^{2}} \frac{\partial^{2}}{\partial t^{2}}-\nabla^{2}\right) p=Q(\boldsymbol{x}, t),
$$

where $Q(\boldsymbol{x}, t)$ is the turbulence quadrupole source,

$$
Q(\boldsymbol{x}, t)=\frac{\partial^{2}\left(\rho_{0} v_{i} v_{j}\right)}{\partial x_{i} \partial x_{j}} .
$$

It is implicitly assumed that the dominant Reynolds stress fluctuations occur in the region $x_{2}>R$ above the roughness elements.

${ }^{\text {a }} R$ is the characteristic height of the roughness elements. 


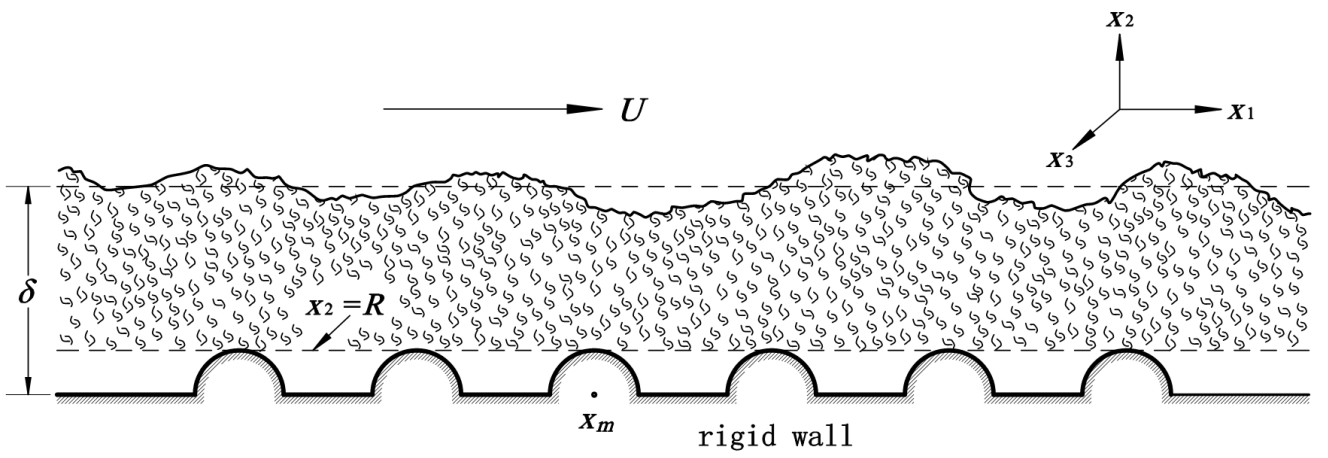

Figure 1. Schematic illustration of the diffraction problem.

Equation (1) is to be solved for the pressure in terms of the Reynolds stress $\rho_{0} v_{i} v_{j}$, subject to the inviscid, high-Reynolds-number, rigid boundary condition

$$
\partial p / \partial x_{n}=0 \quad \text { on } \quad x_{2}=\xi\left(x_{\eta}\right),
$$

where $x_{n}$ is a local coordinate normal to the rough wall. In application, the roughness elements generally protrude beyond the viscous sublayer at the wall into the fully turbulent region of the boundary layer. Even when this is not the case, however, it appears that only a small error is incurred by using inviscid boundary conditions at the wall. ${ }^{5}$ To solve Eq. (1), a Green function $G(\boldsymbol{x}, \boldsymbol{y}, t, \tau)$ is also introduced, which is the solution of Eq. (1) and boundary condition (3), with outgoing wave behavior when the source term $Q$ is replaced by the impulsive point source

$$
\delta(\boldsymbol{x}-\boldsymbol{y}) \delta(t-\tau), \quad x_{2}, y_{2}>\xi\left(x_{\eta}\right) .
$$

Now turbulence of low Mach number generates sound whose characteristic wavelength greatly exceeds the boundary layer thickness $\delta$, and therefore the roughness height $R$. Howe ${ }^{4}$ determined an approximate Green function that satisfies the boundary condition $\partial G / \partial n=0$ on an infinite number of hemispherical bosses of radius $R$, distributed randomly over the plane $y_{2}=0$. In these circumstances he gave the Green function in the form

$$
G(\boldsymbol{x}, \boldsymbol{y}, t, \tau)=\frac{\delta[t-\tau-|\boldsymbol{x}-\boldsymbol{Y}| / c]}{2 \pi|\boldsymbol{x}-\boldsymbol{Y}|},
$$

provided that the far-field point $\boldsymbol{x}$ is situated at a distance much greater than $R$ from the center of the nearest boss, i.e., attention is confined to components of the diffracted field whose length scales are much greater than $R$. In this formula $\boldsymbol{Y}$ is defined by

$$
\begin{aligned}
& Y_{2}=y_{2}, \\
& Y_{\eta}=y_{\eta}+\sum_{m} \mu R^{3}\left(y_{\eta}-x_{m \eta}\right) / 2\left|\boldsymbol{y}-\boldsymbol{x}_{m}\right|^{3}, \quad(\eta=1,3),
\end{aligned}
$$

where $\boldsymbol{x}_{m}=\left(x_{m 1}, 0, x_{m 3}\right)$ is the center of the $m$ th boss on the plane. The factor $\mu$ is to take approximate account of mutual interactions between neighbouring bosses. It is not appreciably different from unity, and is given (to within a relative error of about $4 \%$ ) by

$$
\mu=1 /\left(1+\frac{1}{4} \sigma\right)
$$

where $\sigma=N \pi R^{2}$ is the surface roughness density, i.e. the fractional area of the plane covered by roughness bosses. The function $Y_{\eta}(\boldsymbol{y})$ is identical with the velocity potential describing an ideal incompressible flow in the $\eta$-direction over the rough wall. It satisfies $\partial Y_{\eta} / \partial y_{n}=0$ on $y_{2}=\xi\left(y_{\eta}\right)$ and ensures that $G$ satisfies $\partial G / \partial n=0$ when the acoustic wavelength is much larger than $R$.

Because $p$ and $G$ both satisfy the vanishing normal derivative condition (3), it follows by routine applications of the Green's theorem that the acoustic pressure $p(\boldsymbol{x}, t)$ can be related to the quadrupole source $Q(\boldsymbol{y}, \tau)$ by

$$
p(\boldsymbol{x}, t)=\iint_{-\infty}^{\infty} G(\boldsymbol{x}, \boldsymbol{y}, t, \tau) Q(\boldsymbol{y}, \tau) \mathrm{d}^{3} \boldsymbol{y} \mathrm{d} \tau .
$$




\section{B. Acoustic Spectrum of the Radiated Sound}

The far-field acoustic frequency spectrum will be derived concisely. A different Fourier transform from that of Howe $\mathrm{e}^{4,13}$ is introduced according to the reciprocal relations:

$$
\begin{aligned}
\hat{f}\left(x_{2}, \boldsymbol{\kappa}, \omega\right) & =\iint_{-\infty}^{\infty} f(\boldsymbol{x}, t) e^{-i \boldsymbol{\kappa} \cdot \boldsymbol{x}-i \omega t} \mathrm{~d} x_{1} \mathrm{~d} x_{3} \mathrm{~d} t, \quad \boldsymbol{\kappa}=\left(\kappa_{1}, 0, \kappa_{3}\right), \\
f(\boldsymbol{x}, t) & =\frac{1}{(2 \pi)^{3}} \iint_{-\infty}^{\infty} \hat{f}\left(x_{2}, \boldsymbol{\kappa}, \omega\right) e^{i \boldsymbol{\kappa} \cdot \boldsymbol{x}+i \omega t} \mathrm{~d}^{2} \boldsymbol{\kappa} \mathrm{d} \omega .
\end{aligned}
$$

This ensures that the Fourier transform (9) is consistent with that in the wall pressure spectrum models ${ }^{6}$ and Howe's derivation should be modified accordingly.

In the far field the acoustic power radiated from unit area of the rough wall is equal to the mean acoustic intensity flow, $\langle p v\rangle$, where the angle bracket denotes an ensemble average. The $x_{2}$-component of the perturbation velocity, $v$, is determined from Eq. (8) by making use of the low Mach number approximation to the linearized momentum equation:

$$
\rho_{0} \partial v / \partial t=-\partial p / \partial x_{2}
$$

Following Howe, ${ }^{4}$ the rough-wall acoustic power spectrum $\Pi(\omega)$ is defined as the Fourier transform of $\langle p v\rangle$ with respect to $t$,

$$
\Pi(\omega)=\int_{-\infty}^{\infty}\langle p(\boldsymbol{x}, \tau) v(\boldsymbol{x}, \tau+t)\rangle e^{-i \omega t} \mathrm{~d} t
$$

which expresses the frequency spectrum of the total radiated sound power per unit area of the rough wall. From Eqs. (5)-(8), (10), and (11), $\Pi(\omega)$ can be expressed as

$$
\Pi(\omega)=\frac{N \pi^{2} \mu^{2} R^{6} \omega^{2}}{6 \rho_{0} c^{3}} \int_{-\infty}^{\infty} \Psi(\kappa) e^{-2 \kappa R} \iint_{0}^{\infty} S\left(y_{2}, y_{2}^{\prime} ; \kappa, \omega\right) e^{i\left(\gamma y_{2}-\gamma^{*} y_{2}^{\prime}\right)} \mathrm{d} y_{2} \mathrm{~d} y_{2}^{\prime} \mathrm{d}^{2} \boldsymbol{\kappa},
$$

where the asterisk denotes the complex conjugate. The term $\gamma(\kappa)=\left(k_{0}^{2}-\kappa^{2}\right)^{\frac{1}{2}}$ with $k_{0}=\omega / c$, and the branch cuts are chosen such that $\operatorname{sgn}(\gamma)=\operatorname{sgn}\left(k_{0}\right)$ when $\gamma$ is real, and $\gamma \rightarrow+i \kappa$ as $|\boldsymbol{\kappa}|=\kappa \rightarrow \infty$ on the real axis. $S$ is the cross-spectral density of the turbulence Reynolds stress according to the definition:

$$
S\left(y_{2}, y_{2}^{\prime} ; \boldsymbol{\kappa}, \omega\right)=\frac{1}{(2 \pi)^{3}} \iint_{-\infty}^{\infty}\left\langle\hat{Q}\left(y_{2}, \boldsymbol{\kappa}, \omega\right) \hat{Q}^{*}\left(y_{2}^{\prime}, \boldsymbol{\kappa}^{\prime}, \omega^{\prime}\right)\right\rangle \mathrm{d}^{2} \boldsymbol{\kappa}^{\prime} \mathrm{d} \omega^{\prime}
$$

where $\hat{Q}\left(y_{2}, \boldsymbol{\kappa}, \omega\right)$ is the Fourier transform of the Reynolds stress source $Q(\boldsymbol{y}, \tau)$. Note that $\hat{Q}\left(y_{2}, \boldsymbol{\kappa}, \omega\right)$ is well defined only for $y_{2}>R$, and it vanishes in the region occupied by the roughness bosses. In addition the term $\Psi(\kappa)$ is determined by the average number of roughness bosses in unit area of the plane. The precise value of $\Psi(\kappa)$ is unknown, but for practical purposes use may be made of an interpolation formula given by Howe, ${ }^{4,13}$ namely

$$
\Psi(\kappa)=\frac{\left[1-\sigma J_{1}(2 \kappa R) / \kappa R\right]^{3}}{\left[1+\sigma J_{1}(2 \kappa R) / \kappa R\right]},
$$

where $J_{1}$ is the Bessel function of order unity. This result is exact for $\kappa R=0$, and varies significantly only when $\kappa R$ exceeds unity. Possible discrepancies between interpolated and exact values for large values of $\kappa R$ are of no practical importance because of the accompanying exponential factor $e^{-2 \kappa R}$ in Eq. (12).

Let $P_{R}(\boldsymbol{x}, \omega)$ denote the frequency spectrum of the acoustic pressure fluctuations produced by diffraction by a finite area $A$ of the rough wall, defined such that

$$
\left\langle p^{2}(\boldsymbol{x}, t)\right\rangle=\frac{1}{2 \pi} \int_{-\infty}^{\infty} P_{R}(\boldsymbol{x}, \omega) \mathrm{d} \omega .
$$

If the origin of coordinates is taken at the center of the region $A$, and the observer $\boldsymbol{x}$ is in the acoustic far field from $A$ in a direction specified by the polar angles $(\theta, \phi)$, as illustrated in figure 2, it follows from Eqs. (11), (12), and (15) that ${ }^{4}$

$$
P_{R}(\boldsymbol{x}, \omega)=\frac{A N \pi \mu^{2} R^{6} k_{0}^{2}}{4|\boldsymbol{x}|^{2}} \int_{-\infty}^{\infty} \frac{(\boldsymbol{\kappa} \cdot \tilde{\boldsymbol{n}})^{2} \Psi(\kappa) e^{-2 \kappa R}}{\kappa^{2}} \iint_{0}^{\infty} S\left(y_{2}, y_{2}^{\prime} ; \boldsymbol{\kappa}, \omega\right) e^{i\left(\gamma y_{2}-\gamma^{*} y_{2}^{\prime}\right)} \mathrm{d} y_{2} \mathrm{~d} y_{2}^{\prime} \mathrm{d}^{2} \boldsymbol{\kappa} .
$$




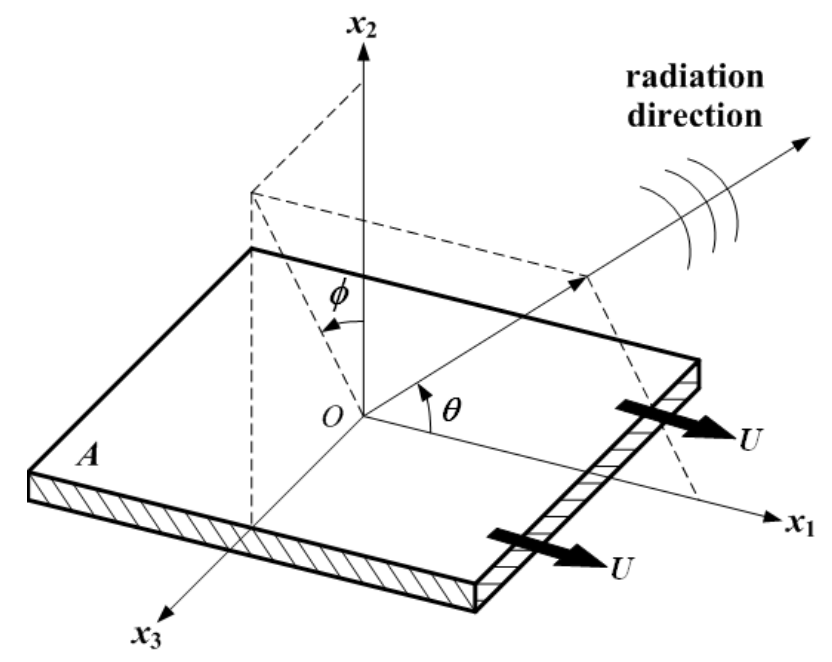

Figure 2. Sound Radiation from a region of area $A$ of the rough wall.

where $\tilde{\boldsymbol{n}}=\boldsymbol{x} /|\boldsymbol{x}|$, and

$$
\boldsymbol{x}=|\boldsymbol{x}|(\cos \theta, \sin \theta \cos \phi, \sin \theta \sin \phi), \quad(0<\theta<\pi,|\phi|<\pi / 2),
$$

is the polar representation of the far-field observer $\boldsymbol{x}$. In this formula, $P_{R}(\boldsymbol{x}, \omega)$ expresses the frequency spectrum of the acoustic pressure at $\boldsymbol{x}$ which is radiated within the solid angle element $\sin \theta \mathrm{d} \theta \mathrm{d} \phi$.

\section{Modeling Turbulence Reynolds Stress Sources}

To complete the determination of the acoustic frequency spectrum $P_{R}(\boldsymbol{x}, \omega)$, it is necessary to model the turbulence Reynolds stress source term, $Q(\boldsymbol{y}, \tau)$. By hypothesis, $Q$ vanishes for $y_{2}<R$, i.e., the principal Reynolds stress noise sources are assumed to be confined to the region $y_{2}>R$. In this case it is convenient to introduce a hypothetical smooth-wall pressure fluctuation $p_{s}(\boldsymbol{x}, t)$ which would be generated on a smooth wall by the same Reynolds stress source. It is determined by solving Eq. (1) with the boundary condition

$$
\partial p_{s} / \partial x_{2}=0 \quad \text { on } \quad x_{2}=0,
$$

and its Fourier transform is given by ${ }^{15}$

$$
\hat{p}_{s}(0, \boldsymbol{\kappa}, \omega)=-\frac{1}{i \gamma(\kappa)} \int_{0}^{\infty} \hat{Q}\left(y_{2}, \boldsymbol{\kappa}, \omega\right) e^{i \gamma y_{2}} \mathrm{~d} y_{2} .
$$

The conventional wavenumber-frequency spectrum $P_{s}(\boldsymbol{\kappa}, \omega)$ of a smooth wall is the Fourier transform of the cross correlation of the pressure in the plane of the wall,

$$
\left\langle\hat{p}_{s}(0, \boldsymbol{\kappa}, \omega) \hat{p}_{s}^{*}\left(0, \boldsymbol{\kappa}^{\prime}, \omega^{\prime}\right)\right\rangle=(2 \pi)^{3} P_{s}(\boldsymbol{\kappa}, \omega) \delta\left(\boldsymbol{\kappa}-\boldsymbol{\kappa}^{\prime}\right) \delta\left(\omega-\omega^{\prime}\right) .
$$

For a rough wall it is necessary to relocate the plane of definition to be $x_{2}=R+0$, just above the roughness bosses. Therefore Eqs. (19) and (20) should be modified as

$$
\begin{gathered}
\hat{p}(R, \boldsymbol{\kappa}, \omega)=-\frac{1}{i \gamma(\kappa)} \int_{R}^{\infty} \hat{Q}\left(y_{2}, \boldsymbol{\kappa}, \omega\right) e^{i \gamma y_{2}} \mathrm{~d} y_{2}, \\
\left\langle\hat{p}(R, \boldsymbol{\kappa}, \omega) \hat{p}^{*}\left(R, \boldsymbol{\kappa}^{\prime}, \omega^{\prime}\right)\right\rangle=(2 \pi)^{3} P(\boldsymbol{\kappa}, \omega) \delta\left(\boldsymbol{\kappa}-\boldsymbol{\kappa}^{\prime}\right) \delta\left(\omega-\omega^{\prime}\right),
\end{gathered}
$$

respectively, to describe the pressure fluctuation and wavenumber-frequency spectrum on a rough wall. The substitution of Eq. (21) into Eq. (22) gives

$$
P(\boldsymbol{\kappa}, \omega)=\frac{1}{|\gamma(\kappa)|^{2}} \iint_{R}^{\infty} S\left(y_{2}, y_{2}^{\prime} ; \boldsymbol{\kappa}, \omega\right) e^{i\left(\gamma y_{2}-\gamma^{*} y_{2}^{\prime}\right)} \mathrm{d} y_{2} \mathrm{~d} y_{2}^{\prime},
$$


where the Reynolds stress cross-spectral density $S\left(y_{2}, y_{2}^{\prime} ; \boldsymbol{\kappa}, \omega\right)$, as defined in Eq. (13), is highly peaked in the neighborhood of the convective ridge of wavenumber-frequency space. Note that $P(\boldsymbol{\kappa}, \omega)$ coincides with $P_{s}(\kappa, \omega)$ when $R=0$.

Substituting Eq. (23) into Eq. (16) and considering the hypothesis that Reynolds stress sources vanishe for $y_{2}<R$, we obtain the acoustic frequency spectrum $P_{R}(\boldsymbol{x}, \omega)$ in a simplified form:

$$
P_{R}(\boldsymbol{x}, \omega)=\frac{A N \pi \mu^{2} R^{6} k_{0}^{2}}{4|\boldsymbol{x}|^{2}} \int_{-\infty}^{\infty} \frac{(\boldsymbol{\kappa} \cdot \tilde{\boldsymbol{n}})^{2}|\gamma(\kappa)|^{2} P(\boldsymbol{\kappa}, \omega) \Psi(\kappa) e^{-2 \kappa R} \mathrm{~d}^{2} \boldsymbol{\kappa}}{\kappa^{2}} .
$$

\section{Wall Pressure Wavenumber-Frequency Spectrum}

In Eq. (24) the rough-wall pressure spectrum $P(\boldsymbol{\kappa}, \omega)$ needs to be specified to evaluate $P_{R}(\boldsymbol{x}, \omega)$. This can be achieved by making use of the available pressure spectrum on a smooth wall. Graham ${ }^{6}$ has drawn a comparison of different empirical models ${ }^{\mathrm{b}}$ for the smooth-wall wavenumber-frequency spectrum of turbulent boundary layer pressures. In his paper the dimensionless wavenumber-frequency spectrum $\tilde{\Phi}_{p}(\boldsymbol{\kappa}, \omega)$ was defined on $x_{2}=0$ in the form:

$$
2 \pi \delta\left(\omega-\omega^{\prime}\right) \tilde{\Phi}_{p}\left(\kappa_{1}, \kappa_{3}, \omega\right)=\frac{\omega^{2}}{U_{c}^{2} \Phi(\omega)} \int_{-\infty}^{\infty}\left\langle p(\boldsymbol{x}+\boldsymbol{\Delta}, \omega) p^{*}\left(\boldsymbol{x}, \omega^{\prime}\right)\right\rangle e^{-i \boldsymbol{\kappa} \cdot \boldsymbol{x}} \mathrm{d} x_{1} \mathrm{~d} x_{3},
$$

where the tilde on $\tilde{\Phi}_{p}$ indicates that it is normalized by $\Phi(\omega) U_{c}^{2} / \omega^{2}$. The typical eddy convection velocity, $U_{c}$, is assumed to be a fixed fraction of the free stream velocity $U$. Both experiments and numerical simulations indicate that $U_{c} \approx 0.5-0.7 U$ with only a weak dependence on the frequency. ${ }^{11} \Phi(\omega)$ is the point pressure frequency spectrum whose curve-fitted formulation will be discussed subsequently. Similar to Eq. (20), Eq. (25) may be rearranged by integrating on both sides, yielding

$$
\tilde{\Phi}_{p}(\boldsymbol{\kappa}, \omega)=\frac{\omega^{2}}{U_{c}^{2} \Phi(\omega)} \frac{1}{|\gamma(\kappa)|^{2}} \iint_{0}^{\infty} S\left(y_{2}, y_{2}^{\prime} ; \boldsymbol{\kappa}, \omega\right) e^{i\left(\gamma y_{2}-\gamma^{*} y_{2}^{\prime}\right)} \mathrm{d} y_{2} \mathrm{~d} y_{2}^{\prime}
$$

For moderately rough surfaces, we assume that the principal features of the wall-pressure spectrum would not differ substantially from those of the pressure spectrum on a smooth wall, especially in the vicinity of the convective peak. ${ }^{12}$ In this case, by comparing the definitions (23) and (26) we can directly relate $\tilde{\Phi}_{p}(\boldsymbol{\kappa}, \omega)$ with $P(\kappa, \omega)$,

$$
P(\boldsymbol{\kappa}, \omega)=\frac{U_{c}^{2}}{\omega^{2}} \Phi(\omega) \tilde{\Phi}_{p}(\boldsymbol{\kappa}, \omega),
$$

provided that the principal contributions to the integral in Eq. (26) are from the region $y_{2}, y_{2}^{\prime}>R$. Therefore by substituting Eq. (27) into Eq. (16) and rearranging, the far-field acoustic pressure frequency spectrum $P_{R}(\boldsymbol{x}, \omega)$ may be expressed in terms of the smooth-wall wavenumber-frequency spectrum $\tilde{\Phi}_{p}(\boldsymbol{\kappa}, \omega)$,

$$
P_{R}(\boldsymbol{x}, \omega)=\frac{A N \pi \mu^{2} R^{6} k_{0}^{2}}{4|\boldsymbol{x}|^{2}} \frac{U_{c}^{2}}{\omega^{2}} \Phi(\omega) \int_{-\infty}^{\infty} \frac{(\boldsymbol{\kappa} \cdot \tilde{\boldsymbol{n}})^{2}|\gamma(\kappa)|^{2} \tilde{\Phi}_{p}(\boldsymbol{\kappa}, \omega) \Psi(\kappa) e^{-2 \kappa R} \mathrm{~d}^{2} \boldsymbol{\kappa}}{\kappa^{2}} .
$$

Note that it is now essential to increase the friction velocity $u_{\tau}$ and boundary layer thickness $\delta$ in the definition of $\tilde{\Phi}_{p}(\boldsymbol{\kappa}, \omega)$ to the corresponding rough-wall values, in order to compensate for the increased surface drag and turbulence production. ${ }^{3,16}$

\section{E. Determinations of $u_{\tau}$ and $\delta$}

The evaluation of $\tilde{\Phi}_{p}(\boldsymbol{\kappa}, \omega)$ in Eq. (28) depends on two boundary layer parameters: 1) the friction velocity $u_{\tau}$; and 2) the boundary layer thickness $\delta$, both of which are influenced by surface roughness. The principal effect of a rough surface is to alter the structure of the boundary layer near the wall thereby increasing the surface skin friction. Hence it is necessary to determine the skin friction on a rough wall.

For fully developed roughness flows (i.e. $k_{s} u_{\tau} / \nu>70$ ) over a flat plate, the effective equivalent height of the roughness particles, $k_{s}$, is very much larger than the thickness of the viscous sublayer. In this case

\footnotetext{
${ }^{\mathrm{b}}$ These empirical models are the Corcos model, ${ }^{7}$ the Efimtsov model, ${ }^{8}$ the Smol'yakov and Tkachenko model, ${ }^{9}$ the Ffowcs Williams model, ${ }^{10}$ the Chase I model, ${ }^{11}$ and the Chase II model, ${ }^{12}$ respectively (see the Appendix for details).
} 
the overall skin friction is independent of $R e_{L}$ and is a function of $L / k_{s}$ only ${ }_{1}^{17}$ where $L$ is the plate length and $R e_{L}=U L / \nu$ is a Reynolds number based on the whole plate. Prandtl and Schlichting ${ }^{18}$ derived an interpolation formula for skin friction based on the sand grain roughness experiments of Nikuradse. ${ }^{19}$ Mills and Hang ${ }^{20}$ compared this formulation with some experimental data sets for fully rough flows over sand grain roughness and found that it gave an average error of 17.5 percent. They attributed the discrepancy to the failure of the formulation to account for the wake component of the mean velocity profile. A new skin friction formula was proposed in the form:

$$
\left.\begin{array}{rl}
c_{f} & =\left[3.476+0.707 \ln \left(x_{1} / k_{s}\right)\right]^{-2.46} \\
C_{F} & =\left[2.635+0.618 \ln \left(L / k_{s}\right)\right]^{-2.57}
\end{array}\right\},
$$

where $c_{f}$ and $C_{F}$ denote the curve-fitted local and overall skin friction coefficients, respectively. This formula gives an average error of 2.7 percent only when compared to the same data and is valid over a wide parameter range. Therefore the friction velocity $u_{\tau}$ can be expressed in terms of $c_{f}$,

$$
u_{\tau}=U \sqrt{c_{f} / 2} .
$$

Following Krogstad et al., ${ }^{21}$ we consider a composite mean velocity profile in a turbulent boundary layer over a rough wall given by

$$
\frac{u}{u_{\tau}}=\frac{1}{\kappa_{0}} \ln \left(\frac{y}{k_{s}}\right)+B+\frac{2 \Pi_{0}}{\kappa_{0}} w\left(\frac{y}{\delta}\right),
$$

where $\kappa_{0} \approx 0.41$ and $B=8.5$ are empirical constants. The wake function $w(y / \delta)$ is expressed as follows

$$
w\left(\frac{y}{\delta}\right)=\frac{1}{2 \Pi_{0}}\left[\left(1+6 \Pi_{0}\right)-\left(1+4 \Pi_{0}\right)\left(\frac{y}{\delta}\right)\right]\left(\frac{y}{\delta}\right)^{2},
$$

where $\Pi_{0}$ represents the strength of the wake. The value of $\Pi_{0}$ for a rough surface is typically larger than for a smooth surface, and it varies with different types of surface roughness. In the present study, $\Pi_{0} \approx 0.45$ has been tentatively applied to the rough surface formed by hemispherical bosses because this value gives the best fit to the velocity profile through hot-wire measurements (see figure 3 ).

The boundary layer thickness $\delta$ on a rough wall is thus solved from Eqs. (29)-(32). If we take $y=\delta$ in Eq. (31), then at this location $u \approx U$ and $w(1)=1$. In this case Eq. (31) is simplified as

$$
\frac{U}{u_{\tau}}=\frac{1}{\kappa_{0}} \ln \left(\frac{\delta}{k_{s}}\right)+B+\frac{2 \Pi_{0}}{\kappa_{0}} .
$$

The only unknown in Eq. (33) is $\delta$ and it can also be expressed in terms of the skin friction coefficient $c_{f}$,

$$
\delta=k_{s} \exp \left(\kappa_{0} \sqrt{2 / c_{f}}-\kappa_{0} B-2 \Pi_{0}\right) .
$$

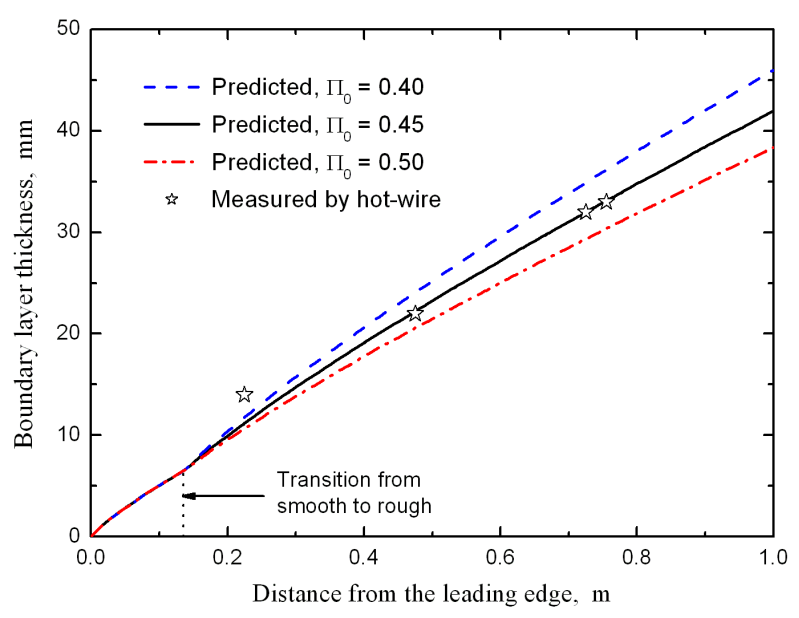

(a) $R=4 \mathrm{~mm}, U=20 \mathrm{~m} / \mathrm{s}$

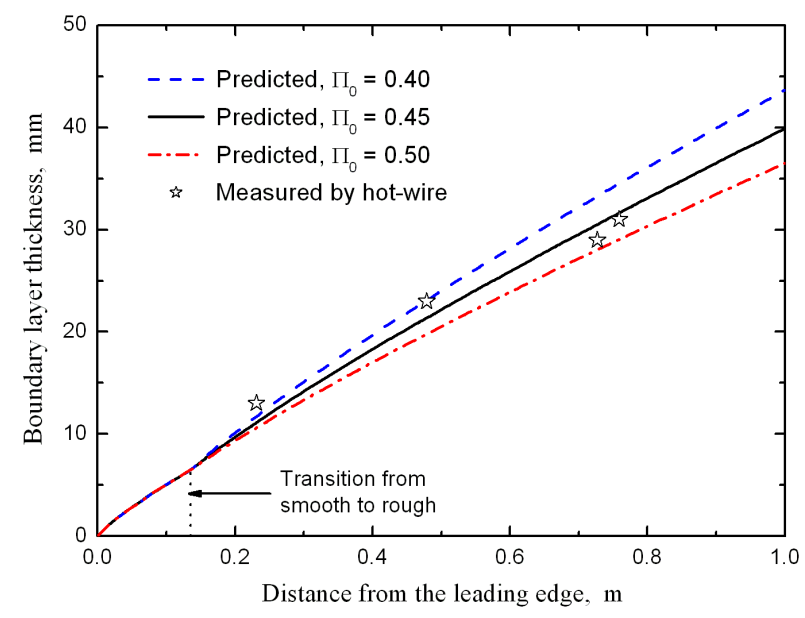

(b) $R=3 \mathrm{~mm}, U=20 \mathrm{~m} / \mathrm{s}$

Figure 3. Comparison between the predicted and measured boundary layer thickness $\delta$ on a rough plate. 
This expression gives the local boundary layer thickness developing on a rough plate. The overall value of $\delta$ for the whole plate, $\bar{\delta}$, can be obtained by substituting $C_{F}$ for $c_{f}$ in Eq. (34).

Figure 3 shows the predicted local boundary layer thickness based on Eq. (34) for two types of roughness elements, i.e. $R=4 \mathrm{~mm}$ and $R=3 \mathrm{~mm}$. The experimental results through hot-wire measurements at several stations along the flat plate are also shown for comparison. As can be seen in figure 3, Eq. (34) plus $\Pi_{0}=0.45$ is able to give very close predictions of the rough-wall boundary layer thickness to the measured values of $\delta$ especially at downstream stations. Noticeable errors occur at upstream stations, but the mean error should be small enough to give an approximate prediction for the overall boundary layer thickness $\bar{\delta}$.

\section{Evaluation of the Rough-Wall Acoustic Pressure Spectrum}

\section{A. Empirical Model by Asymptotic Approximation}

The integral in Eq. (28) will be evaluated herein to obtain the radiated roughness noise in the far field.

As mentioned before the principal components of the boundary layer Reynolds stress occur in the hydrodynamic domain wherein $\kappa=|\boldsymbol{\kappa}| \sim|\omega| / U_{c}$. The wall pressure wavenumber-frequency spectrum $\tilde{\Phi}_{p}(\boldsymbol{\kappa}, \omega)$ is expected to be sharply peaked in the neighbourhood of a "convective ridge" centered on

$$
\kappa_{1} \sim \omega / U_{c}, \quad \kappa_{3}=0,
$$

and the principal contribution to the integral will thus be from the vicinity of the convective peak.

The conventional way to evaluate the integral in Eq. (28) may therefore be obtained by expanding the remaining terms in the integrand about the convective peak and integrating term by term. It is essentially a representation of asymptotic approximation, and may be unable to evaluate the integral with sufficient accuracy. Based on the asymptotic approxiamtion Howe ${ }^{22}$ has proposed an empirical model for the roughwall acoustic frequency spectrum by making use of Chase's empirical formula ${ }^{11}$ of smooth-wall wavenumberfrequency spectrum,

$$
\frac{\Phi_{R}(\boldsymbol{x}, \omega)}{\rho_{0}^{2} u_{\tau}^{3} \delta} \approx \tau_{0} \frac{A \cos ^{2} \theta}{|\boldsymbol{x}|^{2}} \frac{R}{\delta} \frac{u_{\tau}^{2}}{c_{0}^{2}} \frac{\left(\omega R / u_{\tau}\right)^{3}}{\left[1+\beta\left(\omega R / u_{\tau}\right)^{2}\right]^{n / 2}},
$$

where $\tau_{0}, \beta$ and $n$ are empirical coefficients and can be partially estimated by the experimental data of Hersh $^{14}$ on the radiation of sound by sand-roughened pipes of various grit sizes. The best fit to Hersh's data can be achieved by taking

$$
\beta=0.0025, \quad n=11 .
$$

Following Howe ${ }^{22}$ the "roughness parameter" $\tau_{0}$ depends primarily on the spacing of the roughness elements, determined by $\sigma$. When $\sigma \ll 1, \tau_{0} \approx\left(u_{\tau} / U_{c}\right)^{2}(\sigma / \pi)$. However, these values of adjustable coefficients should be regarded as tentative because no directivity information is available from the Hersh data. For this reason, although $\beta$ and $n$ are capable of fixing the shape of the spectrum (see figure 4), it is not possible to derive the absolute levels from Hersh's data.

\section{B. Direct Numerical Integration}

The direct numerical integration is therefore considered instead to evaluate the integral in Eq. (28). It is convenient to introduce the polar representation $(\kappa, \alpha)$ in the wavenumber plane, so that

$$
\kappa_{1}=\kappa \cos \alpha, \quad \kappa_{3}=\kappa \sin \alpha .
$$

Combining Eq. (38) with the definition of the unit vector in the far field

$$
\tilde{\boldsymbol{n}}=\boldsymbol{x} /|\boldsymbol{x}|=(\cos \theta, \sin \theta \cos \phi, \sin \theta \sin \phi)
$$

yields $\boldsymbol{\kappa} \cdot \tilde{\boldsymbol{n}}$ in the polar coordinates,

$$
\boldsymbol{\kappa} \cdot \tilde{\boldsymbol{n}}=\kappa(\cos \alpha \cos \theta+\sin \alpha \sin \theta \sin \phi) .
$$

Therefore the acoustic pressure frequency spectrum $P_{R}(\boldsymbol{x}, \omega)$ becomes

$$
P_{R}(\boldsymbol{x}, \omega)=\frac{A \sigma \mu^{2} R^{4} k_{0}^{2}}{4|\boldsymbol{x}|^{2}} \frac{U_{c}^{2}}{\omega^{2}} \Phi(\omega) D(\theta, \phi),
$$


where $D(\theta, \phi)$ gives the directivity information:

$$
D(\theta, \phi)=I_{1} \cos ^{2} \theta+I_{2} \sin ^{2} \theta \sin ^{2} \phi+2 I_{3} \cos \theta \sin \theta \sin \phi .
$$

In the above expression $I_{1}, I_{2}$, and $I_{3}$ are integrals with respect to the polar coordinates $\kappa$ and $\alpha$,

$$
\begin{aligned}
& I_{1}=\int_{0}^{\infty} \int_{0}^{2 \pi} \cos ^{2} \alpha|\gamma(\kappa)|^{2} \tilde{\Phi}_{p}(\boldsymbol{\kappa}, \omega) \Psi(\kappa) e^{-2 \kappa R} \kappa \mathrm{d} \kappa \mathrm{d} \alpha, \\
& I_{2}=\int_{0}^{\infty} \int_{0}^{2 \pi} \sin ^{2} \alpha|\gamma(\kappa)|^{2} \tilde{\Phi}_{p}(\boldsymbol{\kappa}, \omega) \Psi(\kappa) e^{-2 \kappa R} \kappa \mathrm{d} \kappa \mathrm{d} \alpha, \\
& I_{3}=\int_{0}^{\infty} \int_{0}^{2 \pi} \cos \alpha \sin \alpha|\gamma(\kappa)|^{2} \tilde{\Phi}_{p}(\boldsymbol{\kappa}, \omega) \Psi(\kappa) e^{-2 \kappa R} \kappa \mathrm{d} \kappa \mathrm{d} \alpha .
\end{aligned}
$$

It should be noted that the original infinite double integral over rectangular coordinates in Eq. (28) is now reduced to a series of mixed double integral over polar coordinates, which greatly improves the computation efficiency. The numerical integrations of $I_{1}, I_{2}$, and $I_{3}$ are then carried out by using the five-point Gaussian quadrature with adjustable integration subintervals. Moreover, the infinite $\kappa$-integral can be reduced to a finite integral with sufficient accuracy thanks to the existence of an exponential factor $e^{-2 \kappa R}$ in the integrand. Hence the infinite upper bound in the $\kappa$-integral may be substituted by a positive real number, $\kappa \leqslant 25 / R$, to make the numerical integration practical.

It remains to find a suitable form of the point pressure frequency spectrum $\Phi(\omega)$ in Eq. (41). $\mathrm{Ahn}^{23}$ has approximated a curve fit for the frequency spectrum data in Blake: ${ }^{24}$

$$
\Phi(\omega)=\left(\frac{\tau_{w}^{2} \delta^{*}}{U}\right) \frac{2 \pi 8.28 S h^{*^{0.8}}}{\left[1+4.1 S h^{*^{1.7}}+4.4 \times 10^{-4} S h^{*^{5.9}}\right]},
$$

wherein $\tau_{w}=\rho_{0} u_{\tau}^{2}$ is the mean wall shear stress, and $S h^{*}=\omega \delta^{*} / U$ is the Strouhal number based on the displacement boundary layer thickness $\delta^{*}$. In fact there are a number of forms of $\Phi(\omega)$, e.g. the Efimsov curve fit. ${ }^{8}$ The reason for choosing the form by Ahn is because it is capable of reproducing the features in three different frequency regions as Blake suggested, ${ }^{16}$ whereas the Efimsov curve fit fails to give these features notably at low and high frequencies.

\section{Comparison of Empirical Model with Numerical Method}

A comparison between the empirical model and numerical method is illustrated in figure 4 for the following dimensionless parameter values:

$$
\sigma=0.2, \quad R / \delta=0.01, \quad U_{c} / U=0.6, \quad u_{\tau} / U=0.05, \quad M=0.005 .
$$

These values have been selected to enable the comparison with Howe's empirical model $\Phi_{R}(\boldsymbol{x}, \omega)$, and might be encountered in underwater applications. In these plots the far-field observer is chosen as in the positive $x_{1}$-direction, i.e., $\theta=0$ and $\phi=0$, leading $D(\theta, \phi)$ reduced to $I_{1}$.

The numerical integration of $P_{R}(\boldsymbol{x}, \omega)$ is repeated with different wall pressure spectrum models by Cor$\cos ,{ }^{7}$ Efimtsov, ${ }^{8}$ Smol'yakov and Tkachenko, ${ }^{9}$ and Chase. ${ }^{11,12}$ The Ffowcs Williams model ${ }^{10}$ is rejected, as suggested by Graham, ${ }^{6}$ because its divergent behavior in high wavenumber region does not satisfy the integral requirement:

$$
\frac{1}{(2 \pi)^{2}} \iint_{-\infty}^{\infty} \tilde{\Phi}_{p}\left(\kappa_{1}, \kappa_{3}, \omega\right)\left(\frac{U_{c}}{\omega}\right)^{2} \mathrm{~d} \kappa_{1} \mathrm{~d} \kappa_{3}=1 .
$$

The curves in figure 4 represent the variations of $\Phi_{R}(\boldsymbol{x}, \omega)$ and $P_{R}(\boldsymbol{x}, \omega)$ as functions of the dimensionless frequency $\Omega=\omega R / u_{\tau}$. Hersh's data ${ }^{14}$ for sand-roughened pipes of various grit sizes are also shown as

scatter points. No comparison of the absolute levels of the predicted and measured noise is possible due to the unknown effects of acoustic refraction by the free jet shear layers downstream of the nozzle exit. ${ }^{13}$ Accordingly, the heights of the curves have been adjusted to yield the best fit to the data points.

As shown in figure 4, the shape of the empirical spectrum $\Phi_{R}(\boldsymbol{x}, \omega)$ fits the experimental data very well by taking the coefficient values in Eq. (37). Moreover, the comparison between the empirically and numerically 


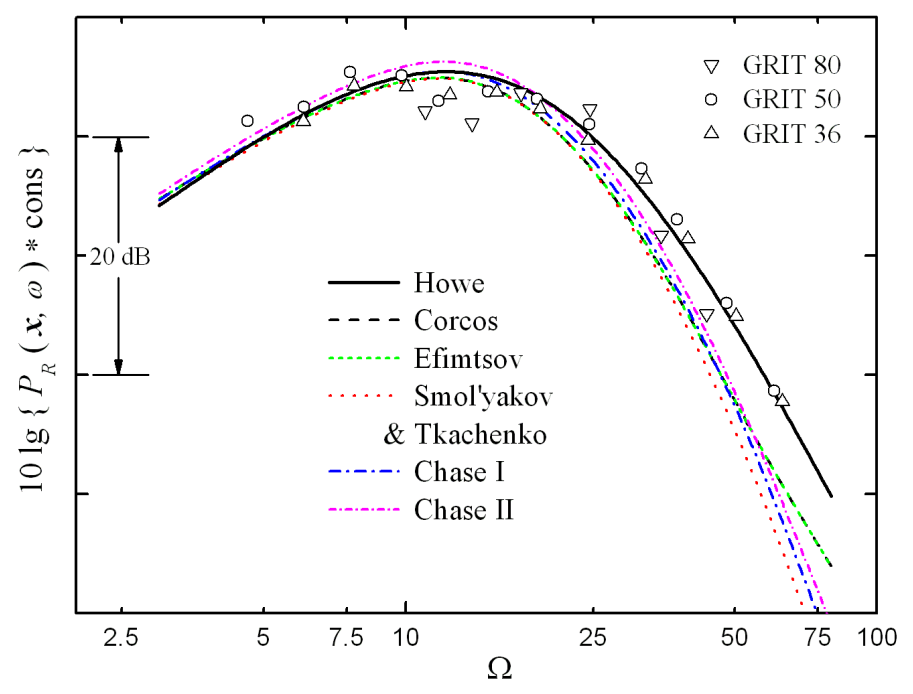

Figure 4. Roughness noise spectra predicted by the empirical model and numerical integration together with the experimental data by Hersh.

predicted roughness noise spectra reveals remarkable correlation. Both $\Phi_{R}(\boldsymbol{x}, \omega)$ and $P_{R}(\boldsymbol{x}, \omega)$ peak around $\Omega \approx 13$ and decay rapidly at higher frequencies. The relatively abrupt fall-off of $P_{R}(\boldsymbol{x}, \omega)$ is probably due to the features of $\Phi(\omega)$ specified in Eq. (44). In addition, the numerically predicted spectra obtained from different empirical models of $\tilde{\Phi}_{p}(\boldsymbol{\kappa}, \omega)$ agree with one another very well. In this dimensionless frequency range $(3 \leq \Omega \leq 80)$, the Efimtsov model reduces to the Corcos model and thus their curves coincide, predicting levels comparable to other models except when $\Omega>60$.

\section{Experimental Validation}

\section{A. Experimental Setup}

The experiments were performed in an open jet of a low-speed wind tunnel to measure the radiated sound from a rough plate. A schematic of the experimental setup is shown in figure 5 . The wind tunnel has a inner cross-section of $0.586 \mathrm{~m} \times 0.35 \mathrm{~m}$ and a velocity range of $0-31.0 \mathrm{~m} / \mathrm{s}$; it was lined with plastic foams on the inner wall to reduce the fan noise and flow noise traveling inside the tunnel. A flat plate was placed in the center of the open jet with the plate plane perpendicular to the ground. The plate surface was partially roughened in a $0.64 \mathrm{~m} \times 0.64 \mathrm{~m}$ square region by rigid hemispherical plastic beads placed in parallel rows. Each row spans the entire height of the rough region. This was achieved by drilling a number of hemispherical holes into four modeling boards with spherical beads glued into these holes, and then mounting these boards flush in a square recess milled into the flat plate. Two different surface roughness conditions were examined: 1) $R=4 \mathrm{~mm}, \sigma=0.50$; and 2) $R=3 \mathrm{~mm}, \sigma=0.44$; together with a smooth surface for comparison. The values of both $R$ and $\sigma$ were chosen comparatively high to ensure that the roughness noise could be detected by the microphones.

The leading edge of the flat plate was carefully streamlined to reduce sound scattering effects due to flow separation at a bluff-headed nose. The boundary layer was tripped using four layers of tape cut in sawtooth form with $0.13 \mathrm{~mm}$ in thickness and $1.5 \mathrm{~mm}$ in width. The tape was placed $4 \mathrm{~cm}$ from the leading edge over the entire span of the plate. This encourages a fully developed turbulent boundary layer close to the leading edge. Also, the rough region was located at $0.34 \mathrm{~m}$ from the leading edge of the plate to satisfy the assumption that the roughness elements are contained entirely within the boundary layer.

In the first setup of acoustic measurements, the radiated sound from the rough plate was detected by four $1 / 2$ " free-field condenser microphones as marked in figure 5. In a duration time of $60 \mathrm{~s}$ the acoustic signal were acquired at a sampling frequency of $65.5 \mathrm{kHz}$ in the frequency range $7 \mathrm{~Hz}-25.6 \mathrm{kHz}$. The coordinates are also indicated in the schematic with the origin at the center of the rough region. The four condenser microphones were arranged in a $0.16 \mathrm{~m} \times 0.16 \mathrm{~m}$ square array on the plane $x_{3}=0$ and axisymmetric to the plane $x_{1}=0$ with microphones M1 \& M2 upstream and M3 \& M4 downstream. The spacing between 


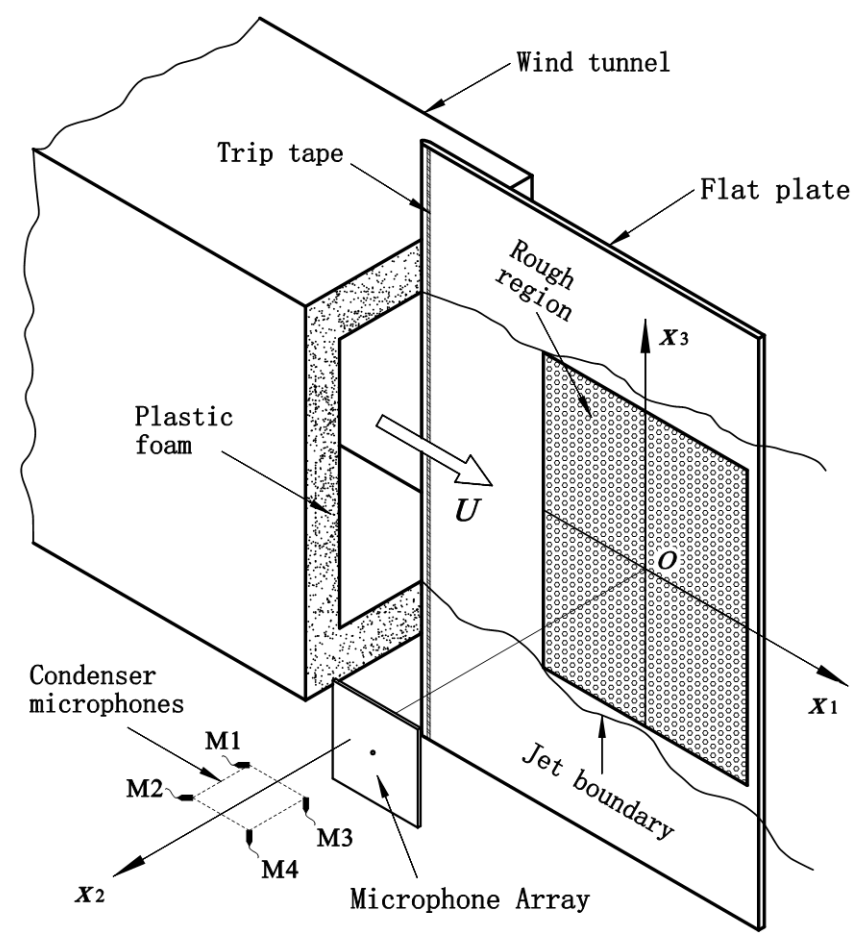

Figure 5. Schematic of the experimental setup.

each two microphones, $0.16 \mathrm{~m}$, is based on the preliminary numerical prediction which indicated a spectral peak around $2 \mathrm{kHz}$. Therefore the microphone spacing should be adjusted as less than or close to one wavelength of $2 \mathrm{kHz}, c a .0 .17 \mathrm{~m}$, to obtain the best cross correlation at this peak frequency. Furthermore, to reduce unwanted turbulence fluctuations around the microphones (wind noise), it is important to put the microphones out of the jet boundary, and hence the distance between the plate plane and front microphones M1 \& M3 was set as $0.6 \mathrm{~m}$.

In the second setup, a phased microphone array was utilized to localize the sound sources in the rough region. The advantage of a phased microphone array lies in its improved signal-to-noise ratio; hence noise sources below the background noise can be identified. The acoustic array consists of a total of 48 microphones which are located on optimized concentric circles and flush mounted in a rigid board. As shown in figure 5, the array board is parallel to the plate plane with a relatively short distance of $0.47 \mathrm{~m}$ to give better resolution. The center of the array board was located on the plane $x_{3}=0$, too, but with a offset of $4 \mathrm{~cm}$ downstream. In the acoustic tests, the data from the array microphones were synchronously measured at a sampling frequency of $120 \mathrm{kHz}$ and a measurement duration of $60 \mathrm{~s}$. Then the data were post-processed and source maps in $1 / 3$ octave bands were calculated by a beamforming of the data. Herein the "beamforming" means an algorithm for the phased microphone array, and the beamforming code for the post-processing is a frequency-domain beamforming.

\section{B. Modifications of the Numerical Scheme}

For the experimental setup illustrated in figure 5, the numerical scheme (41) should be accordingly modified to enable the comparison of the numerical prediction with experimental results.

Firstly, the nonuniform directivity function $D(\theta, \phi)$ for sound radiated from different parts of the rough region should be taken into account as the far-field distance $|\boldsymbol{x}|$ and the dimensions of the rough area $A$ are now at approximate length scales. In this case the rough area $A$ should be divided into a number of equal subareas $A_{j}, j=1, \ldots, N_{A}$; then apply the numerical integration for the roughness noise radiations from each subarea, and add them up to obtain the total radiation from the whole rough region. For the present experimental setup, $|\boldsymbol{x}| \gg \sqrt{A_{j}}$ can be satisfied if the number of subareas $N_{A}>12$ is taken.

Secondly, the cross spectra were used for the measured noise spectra to discard the interference of uncorrelated noise signals. Thus the numerical prediction should also consider the cross correlation between 


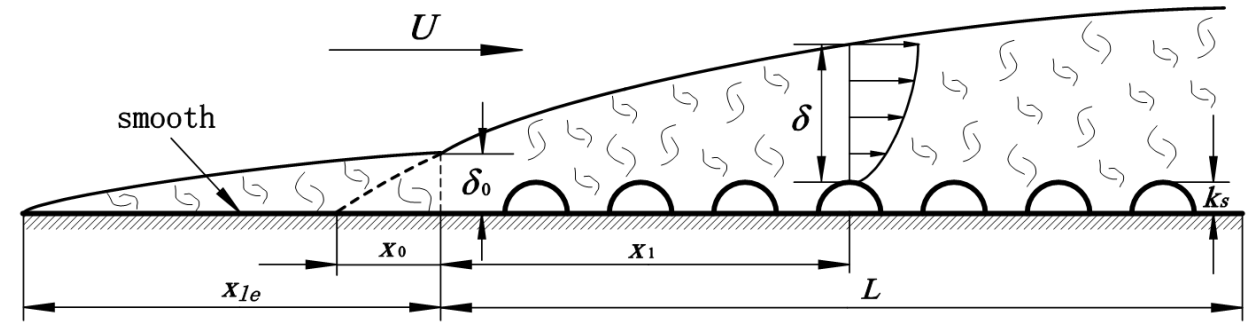

Figure 6. The rough region located at a distance from the leading edge.

two microphones. This can be approximated by taking the average far-field distance $|\overline{\boldsymbol{x}}|$ and directivity angles $(\bar{\theta}, \bar{\phi})$ of these two microphones with an extra term $e^{-i k_{0}\left(\left|\boldsymbol{x}_{1}\right|-\left|\boldsymbol{x}_{2}\right|\right)}$ in Eq. (41).

Taking into these two factors, the numerical scheme (41) becomes

$$
P_{R}(\overline{\boldsymbol{x}}, \omega)=\sum_{j=1}^{N_{A}} \frac{A_{j} \sigma \mu^{2} R^{4} k_{0}^{2}}{4|\overline{\boldsymbol{x}}|^{2}} \frac{U_{c}^{2}}{\omega^{2}} \Phi(\omega) D\left(\bar{\theta}_{j}, \bar{\phi}_{j}\right) e^{-i k_{0}\left(\left|\boldsymbol{x}_{1}\right|-\left|\boldsymbol{x}_{2}\right|\right)},
$$

which expresses the cross spectra of the roughness noise radiated from a total of $N_{A}$ rough subareas.

It should also be noted that in figure 5 the rough region starts at $0.34 \mathrm{~m}$ from the leading edge, whereas it is assumed in the skin friction formula (29) that the surface is roughened on the whole plate. This contradiction can be removed by a correction to the length of the rough region. As shown in figure 6 , the boundary layer grows along the front smooth surface until it achieves a thickness $\delta_{0}$ at the edge of the rough region. This boundary layer thickness can be calculated by means of the $1 / 9$ power law for smooth walls, ${ }^{25}$

$$
\delta_{0}=0.36 x_{l e} / R e_{l e}^{1 / 6},
$$

where $R e_{l e}=U x_{l e} / \nu$ is a Reynolds number based on the distance $x_{l e}$ from the leading edge. In another way $\delta_{0}$ can be assumed as produced by a hypothetical extension $x_{0}$ of the rough region. Substituting this value of $\delta_{0}$ into Eq. (34) and combining Eq. (29) gives the correction term

$$
x_{0}=k_{s} \exp \left[\left(c_{f 0}^{-1 / 2.46}-3.476\right) / 0.707\right]
$$

where $c_{f 0}$ is the local skin friction coefficient at $x_{l e}$,

$$
c_{f 0}=2 /\left[\ln \left(\delta_{0} / k_{s}\right)+\kappa_{0} B+2 \Pi_{0}\right]^{2} .
$$

Therefore the length scales in the skin friction formula (29), i.e. $x_{1}$ and $L$, are corrected to $x_{1}+x_{0}$ and $L+x_{0}$ respectively for the rough region not starting from the leading edge.

\section{Results and Discussion}

The sound pressure spectra for the rough and smooth plates measured by the $1 / 2$ " condenser microphones are shown in figure 7 and compared with the numerically predicted roughness noise spectra using different wavenumber-frequency spectrum models. The acoustic measurements were performed for a series of flow velocities, $U=10,15, \ldots, 30 \mathrm{~m} / \mathrm{s}$, but only the experimental results for $U=30 \mathrm{~m} / \mathrm{s}$ are presented because the surface roughness generated noise is too quiet at lower flow velocities to reveal evident differences between the noise spectra of rough and smooth plates. The cross spectra results are presented for the microphone pair, M3 \& M4, because it showed the greatest signal to noise. The experimental data were processed in narrow band and divided by the bandwidth $\Delta f=8 \mathrm{~Hz}$.

As shown in figure 7 , background noise dominates at most of the frequencies including noticeable peaks at about $3 \mathrm{kHz}$ and $4.5 \mathrm{kHz}$ which are from the motor and fan of the wind tunnel. However, the measured noise spectra of the rough plates are above that of the smooth plate between 1-2.5 kHz, making the roughness noise detectable from the noise of the smooth plate. This appears to be confirmed by the fact that the rough-plate spectra are less fluctuating than the that of the smooth plate in this frequency range, which suggests that they contain statistically steady sources actively radiating sound. 


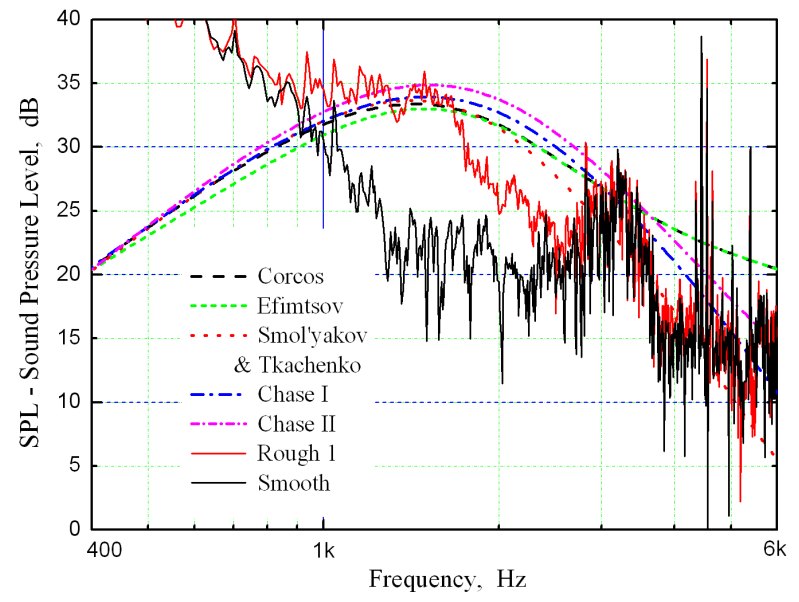

(a) Rough $1, R=4 \mathrm{~mm}, \sigma=0.50$

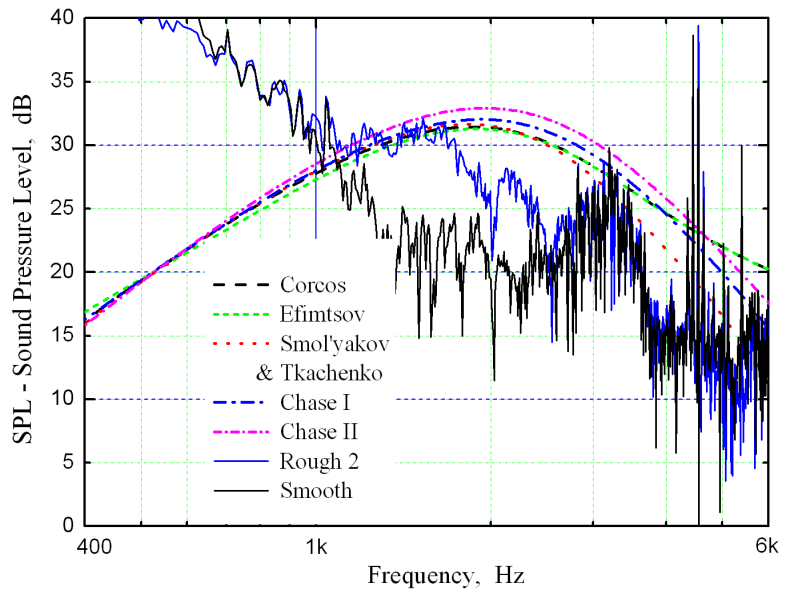

(b) Rough $2, R=3 \mathrm{~mm}, \sigma=0.44$

Figure 7. Comparison between experimental and predicted roughness noise spectra. Smooth-wall experimental results are shown for comparison.

The comparison between the measured and predicted roughness noise spectra exhibits encouraging agreement. The numerical method is capable of giving absolute predictions for the radiated roughness noise at approximate levels of the experimental results in the frequency range 1-1.7 kHz, although the difference becomes notable at higher frequencies. Namely, the major disagreement lies in the relatively high peak
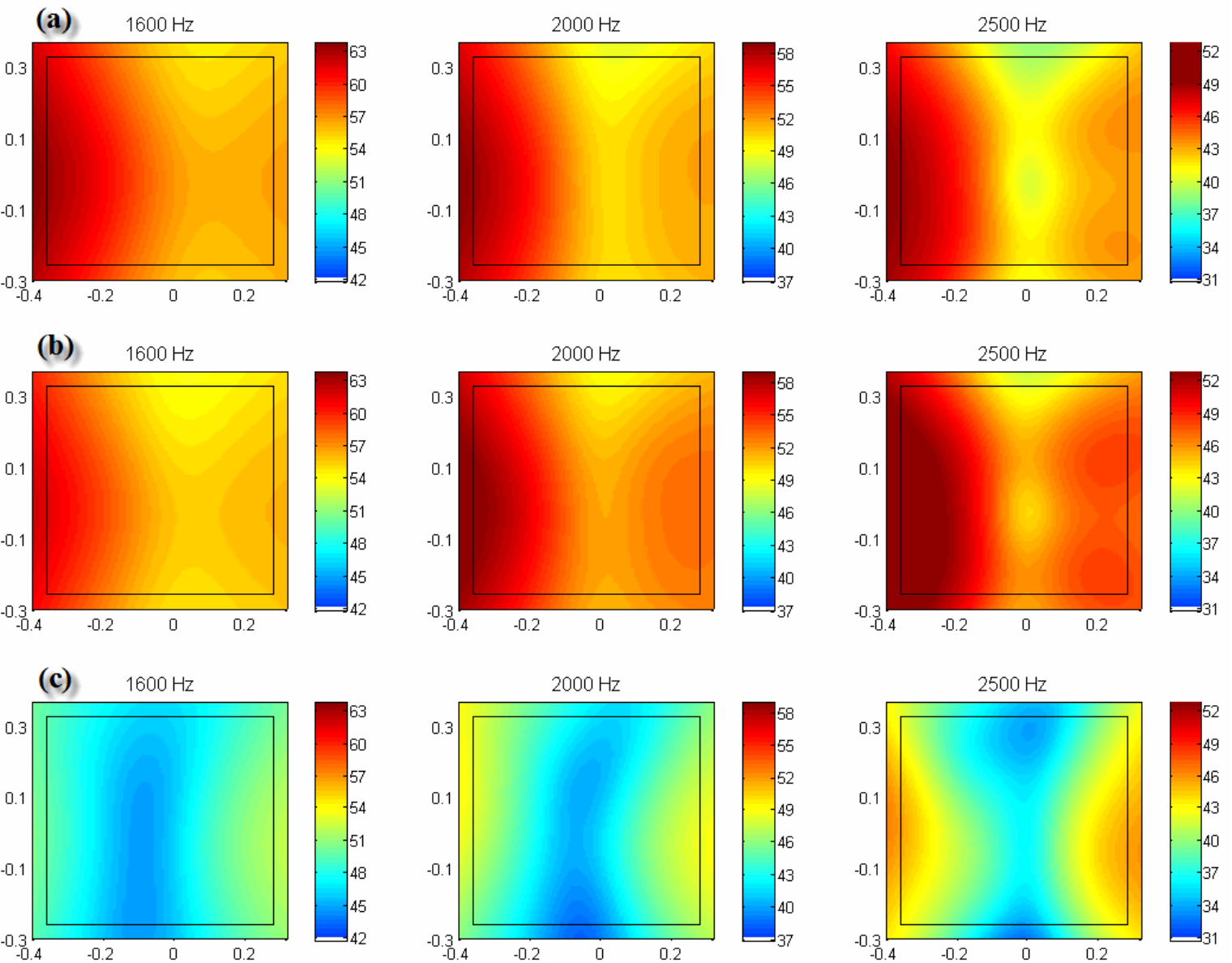

Figure 8. Source maps for the rough and smooth plates: (a) Rough 1, (b) Rough 2, (c) Smooth. 
frequency and slow decay of the predicted spectra. Comparing the two roughness elements, one notices that the smaller one displays a lower spectral peak, though not very distinct, by about $5 \mathrm{~dB}$ at a slightly higher frequency. Moreover, it is shown that different wavenumber-frequency spectrum models produce approximate roughness noise spectra in which the Smol'yakov and Tkachenko model gives the closest fit to the measured spectra at frequencies higher than $1.7 \mathrm{kHz}$.

On the basis of the experimental data through the $1 / 2$ " condenser microphones, it is worthwhile to discover the possible sound sources in the rough region in the frequency range 1-2.5 $\mathrm{kHz}$ wherein the roughness noise is detectable from the noise of the smooth plate. Figure 8 illustrates the source maps obtained by the phased microphone array for the rough and smooth plates at the center frequencies of $1 / 3$ octave bands, i.e. 1.6, 2.0 and $2.5 \mathrm{kHz}$, respectively. The source strengths of the three maps at the same frequency are transformed to the same reference scales to enable the comparison. The inside dark squares outline the boundary of the rough region and the flow direction is from the left to the right.

As can be seen from the source maps, the source strengths of the two rough plates appear very similar, with the maximum source region near the front edge of the rough region. This can be explained as that the boundary layer is gradually growing along the plate and thus the roughness elements downstream are further away from the turbulent sources. Nevertheless, the source maps of the smooth plate indicate remarkably lower source strengths than those of the rough plates, especially at the center of the nominal rough region where the source strengths are at least $8-10 \mathrm{~dB}$ below those of the roughness noise sources. This difference is also in accordance with the comparison of noise spectra in figure 7.

\section{Numerical Prediction and Analysis}

\section{A. Roughness Noise from an Aircraft Wing}

The comparisons of both the spectral shape and absolute level of the predicted roughness noise with both the empirical model and experimental results have provided some validation of the diffraction theory of turbulent boundary-layer generated roughness noise. On this basis the diffraction theory and numerical scheme were employed to investigate the effects of surface roughness on airframe noise. Herein the far-field roughness noise generated by an aircraft wing will be numerically predicted and compared with the corresponding trailing edge (TE) noise.

The roughness noise prediction is based roughly on the dimensions of the wing of a Boeing 757 sized aircraft. The wing has been approximately modeled as a flat plate with a rough estimate of the following dimensional parameters

$$
L_{c} \approx 5 \mathrm{~m}, \quad L_{s} \approx 16.5 \mathrm{~m}, \quad A=L_{c} \cdot L_{s} \approx 82.5 \mathrm{~m}^{2},
$$

where $L_{c}$ and $L_{s}$ are the equivalent chord and span, respectively, and $A$ is the equivalent area of the wing. The aircraft noise is most annoying to the people outside the airport perimeter in a typical built-up area when the airplane is approaching to or taking off from the airport. In this case the typical Mach number $M$ of the free stream velocity and far-field observer may be taken as

$$
M=U / c_{0}=0.2, \quad|\boldsymbol{x}|=500 \mathrm{~m}, \quad \theta=\pi / 4, \quad \phi=0 .
$$

Furthermore, it is necessary to evaluate the ensemble average $\left\langle p^{2}(\boldsymbol{x}, t)\right\rangle$ of the far-field acoustic pressure by integrating the frequency spectrum $P_{R}(\boldsymbol{x}, \omega)$ over the audio frequency range, $f \in[20 \mathrm{~Hz}-20 \mathrm{kHz}]$. Aweighting ${ }^{26}$ is also taken into account in the integration to yield a more accurate noise prediction. Provided that $P_{R}(\boldsymbol{x}, \omega)$ is an even function and $\omega=2 \pi f$, the representation of $\left\langle p^{2}(\boldsymbol{x}, t)\right\rangle$ in Eq. (15) becomes

$$
\left\langle p^{2}(\boldsymbol{x}, t)\right\rangle=\frac{1}{\pi} \int_{40 \pi}^{4 \times 10^{4} \pi} 10^{C_{A} / 10} P_{R}(\boldsymbol{x}, \omega) \mathrm{d} \omega,
$$

where $C_{A}$ is the A-weighting factor (dBA) dependent on the frequency. Exact values of $C_{A}$ are not available for all definite frequencies; hence interpolation is used if necessary.

Finally, the sound pressure level (SPL) of the rough noise spectrum is obtained by comparing $P_{R}(\boldsymbol{x}, \omega)$ with the reference sound intensity level

$$
\mathrm{SPL}=10 \lg \left[\frac{P_{R}(\boldsymbol{x}, \omega)}{4 \times 10^{-10} \mathrm{~Pa}^{2}}\right], \mathrm{dB} .
$$


Similarly, the overall sound pressure level (OASPL) is given by

$$
\text { OASPL }=10 \lg \left[\frac{\left\langle p^{2}(\boldsymbol{x}, t)\right\rangle}{4 \times 10^{-10} \mathrm{~Pa}^{2}}\right], \mathrm{dBA}, \quad f \in[20 \mathrm{~Hz}-20 \mathrm{kHz}] .
$$

For comparison, the corresponding TE noise was also calculated for both SPL and OASPL. However, the TE noise prediction was based on the same dimensions of the aircraft wing but with a smooth surface. This is because the surface roughness is generally not taken into account in the traditional estimate of airframe noise, and hence its effects will be more explicit if the roughness noise is compared with the traditionally predicted TE noise. Moreover, to be consistent with the roughness noise prediction, the TE noise is also predicted on the basis of an empirical model by Howe ${ }^{22}$ with Chase's empirical formula ${ }^{11}$ of wall pressure spectrum. The directivity function in this model has been taken as 1 to obtain the maximum sound radiation.

\section{B. Parametric Study}

A parametric study was firstly carried out to investigate the effects of two important parameters, roughness height $R$ and roughness density $\sigma$, on the roughness noise from a Boeing 757 sized aircraft wing. For simplicity, the numerical integration was based on the Smol'yakov and Tkachenko model only.

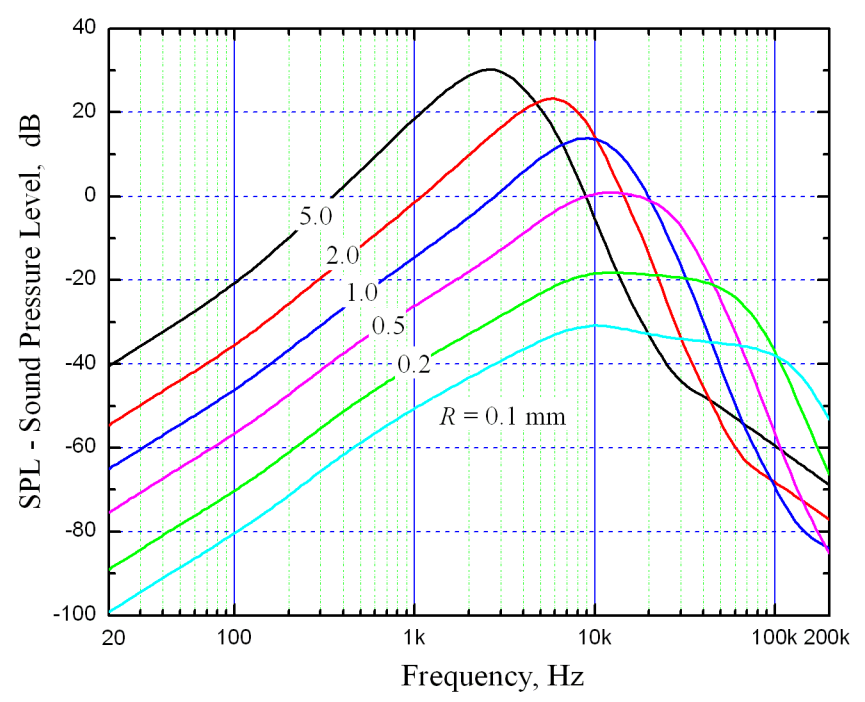

Figure 9. Effects of roughness height $R$ on predicted roughness noise spectra, $\sigma=0.85$.

In figure 9, the roughness noise spectra for different values of $R$ are compared in a wide frequency range, $20 \mathrm{~Hz}-200 \mathrm{kHz}$, with the value of $\sigma$ fixed to 0.85 . As $R$ decreases from $5.0 \mathrm{~mm}$ the spectral peak shifts to progressively higher frequencies with diminishing SPL until $R=0.1 \mathrm{~mm}$. Although there are no evident peaks for $R<0.1 \mathrm{~mm}$, remarkable reduction of SPL is still in existence. In addition, figure 9 implies that the peak frequency of the roughness noise spectrum is determined to a great extent by the scale of roughness elements. This might be explained as that the wavelength $\lambda$ of the acoustic frequency at which the strongest scattering occurs should be at the approximate length scale as the roughness height $R$. For this reason the effect of surface roughness is negligible when $R$ is small compared with $\lambda$, i.e. $k_{0} R \ll 1$ for "compact" roughness elements. This accounts for the low spectral levels for roughness elements with small heights or at low frequencies.

Furthermore, the dependence of roughness noise OASPL on roughness height $R$ for various values of $\sigma$ is depicted in figure 10(a). It is shown evidently that the parameter $R$ has significant effects on the roughness noise OASPL. Take $\sigma=0.05$ for example, as $R$ increases from $0.1 \mathrm{~mm}$, the roughness noise OASPL increases from $9.3 \mathrm{dBA}$ monotonically and then begins to level off at $R=2.5 \mathrm{~mm}$ until it approaches $49.3 \mathrm{dBA}$ for very large roughness heights $(R \geqslant 5 \mathrm{~mm})$.

On the other hand, roughness density $\sigma$ is another important parameter for the roughness noise. As shown in figure 10(a), larger values of $\sigma$ usually generate louder roughness noise for large roughness elements $(R \geqslant 2.0 \mathrm{~mm})$. However, it is not always the case. This is illustrated more obviously in figure 10(b) in which 


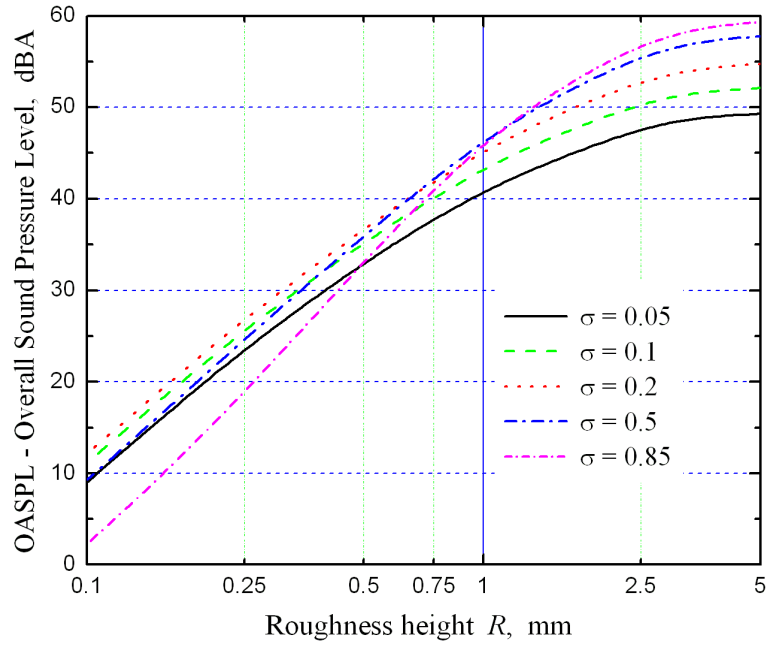

(a) OASPL vs. $R$

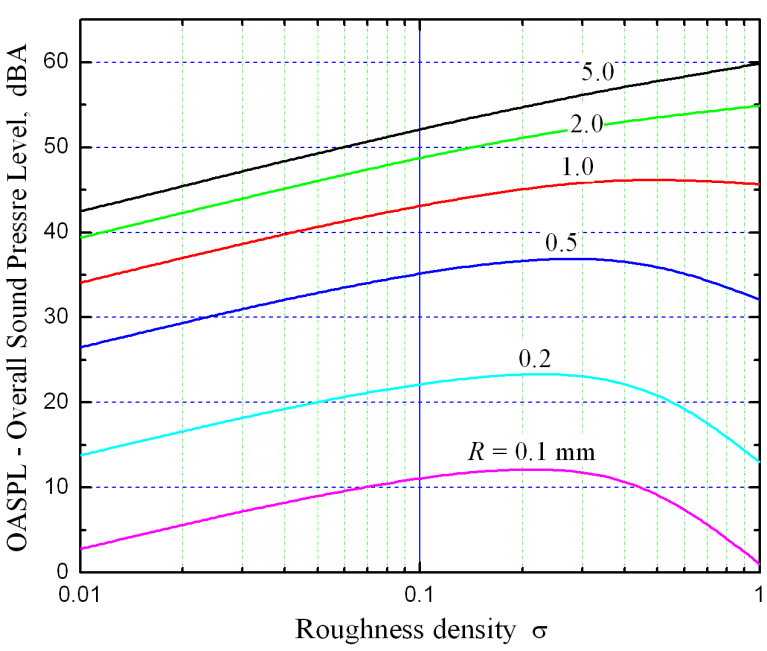

(b) OASPL vs. $\sigma$

Figure 10. Effects of both roughness height $R$ and roughness density $\sigma$ on predicted roughness noise OASPL.

for $R \leqslant 1.0 \mathrm{~mm}$ the roughness noise OASPL increases slowly with $\sigma$, attains a maximum at $\sigma_{p}$, and then decays. The peak value $\sigma_{p}$ shifts, as $R$ increases, to a larger value of $\sigma$ with higher OASPL. This is because that for larger values of $\sigma$ than $\sigma_{p}$ the wall appears gradually "smoother" as roughness elements become progressively compacter. But this effect will be counteracted to some extent by larger roughness heights $(R \geqslant 2.0 \mathrm{~mm})$ because the absolute space between the roughness elements increases with $R$ even for a very dense distribution.

Nevertheless, the effect of roughness density $\sigma$ on roughness noise prediction is not as significant as that of roughness height $R$. For instance, the OASPL variation in figure 10(b) is at most $17.4 \mathrm{dBA}$ from $\sigma=0.01$ to 1.0 for the case $R=5.0 \mathrm{~mm}$, although such a large roughness density of 1.0 is not achievable in practice, which is considerably less than the OASPL increase of at least $40 \mathrm{dBA}(\sigma=0.05)$ from $R=0.1 \mathrm{~mm}$ to $5.0 \mathrm{~mm}$ in figure $10(\mathrm{a})$.

\section{Test Cases}

Based on the parametric study, three idealized test cases were considered to approximately quantify the possible roughness noise from the wing of a Boeing 757 sized aircraft:

(1) Rough surfaces formed by rivets, ribs, joints, etc.;

(2) Rough surfaces formed by environmental contamination;

(3) Dip-galvanized metal surfaces.

The relative parameters are listed in table 1 . As previously mentioned, the values of $u_{\tau}$ and $\delta$ for Cases (1) and (2) have been increased substantially due to the increased surface drag and turbulence production. However, because of the very small roughness height, the surface of Case (3) appears more like a smooth wall with similar parameter values.

Table 1. Test case parameters.

\begin{tabular}{cccccccc} 
Case $^{*}$ & $R, \mathrm{~mm}$ & $\sigma$ & $C_{F}$ & $u_{\tau}, \mathrm{m} / \mathrm{s}$ & $u_{\tau} / U$ & $\bar{\delta}, \mathrm{cm}$ & $R / \bar{\delta}$ \\
\hline$(1)$ & 5.0 & 0.05 & 0.0060 & 3.72 & 0.055 & 11.28 & 0.0443 \\
$(2)$ & 0.5 & 0.85 & 0.0036 & 2.90 & 0.043 & 9.38 & 0.0053 \\
$(3)$ & 0.152 & 0.85 & 0.0029 & 2.59 & 0.038 & 8.91 & 0.0017
\end{tabular}

${ }^{*}$ The corresponding values of $C_{F}, u_{\tau} / U$, and $\bar{\delta}$ for smooth walls can be characterized as $0.0027,0.037$, and $6.07 \mathrm{~cm}$, respectively. 
Furthermore, the roughness elements in these test cases are generally not perfect hemispheres, and $R$ herein corresponds to the equivalent roughness height. The value of $\sigma$ varies with the different rough surfaces. It is reasonable to take $\sigma$ as small as 0.05 for an aerofoil surface with a sparse distribution of rivets, ribs, joints, etc., whereas for the other cases the roughness elements are assumed distributed very densely over the aerofoil surface. Following Howe, ${ }^{4}$ the maximum value of roughness density, $\sigma_{\max } \sim 0.91$, occurs for a hexagonal close packing, but this would not be representative for a purely random distribution. The value of $\sigma$ for a square close packing is $\sigma_{s q} \sim 0.78$. Hence the mean value of $\sigma_{\max }$ and $\sigma_{s q}, c a .0 .85$, is tentatively used as the practical maximum $\sigma$ in Cases (2) and (3).

The predicted roughness noise spectra for the three test cases are depicted in figure 11, and compared with corresponding TE noise spectra. Similar to figure 4, different empirical models of wavenumber-frequency spectrum produce approximate roughness noise spectra, especially around the peaks where all these curves almost coincide with one another. As can be seen in figure 11, the TE noise spectra are significant only in the low frequency region. They always peak around very low frequencies, $c a$. $65 \mathrm{~Hz}$, then decay monotonically with increasing frequencies. The roughness noise spectra, however, can be nearly ignored in the low frequency region but achieve their peaks at very high frequencies. This tends to confirm that the roughness noise can be comparable to or even more significant than the corresponding TE noise provided with sufficiently high frequencies.

For Case (1), a distinct spectral peak of the roughness noise occurs around $2 \mathrm{kHz}$ and is at the approximate level as that of the TE noise. However for Cases (2) and (3), the former becomes obviously lower than the latter due to the diminishing size of roughness elements. Nevertheless, the roughness noise spectra of Case (2)

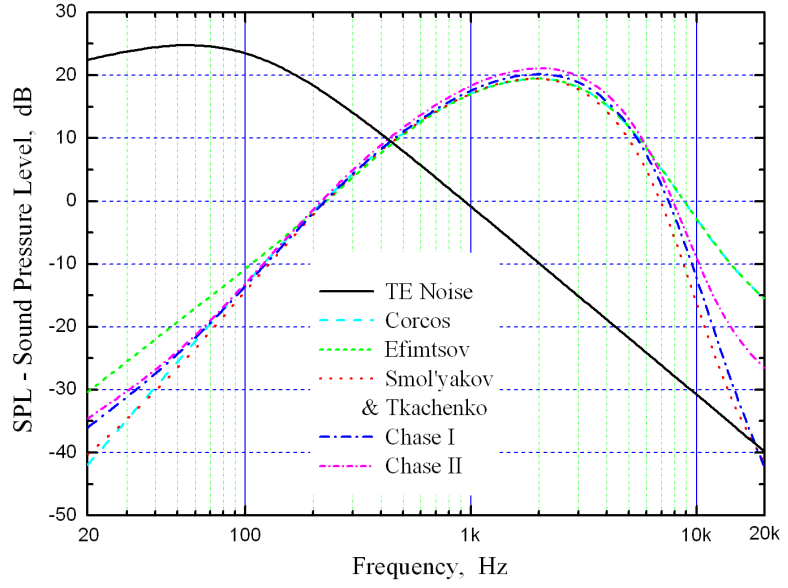

(a) Case (1), $R=5 \mathrm{~mm}, \sigma=0.05$

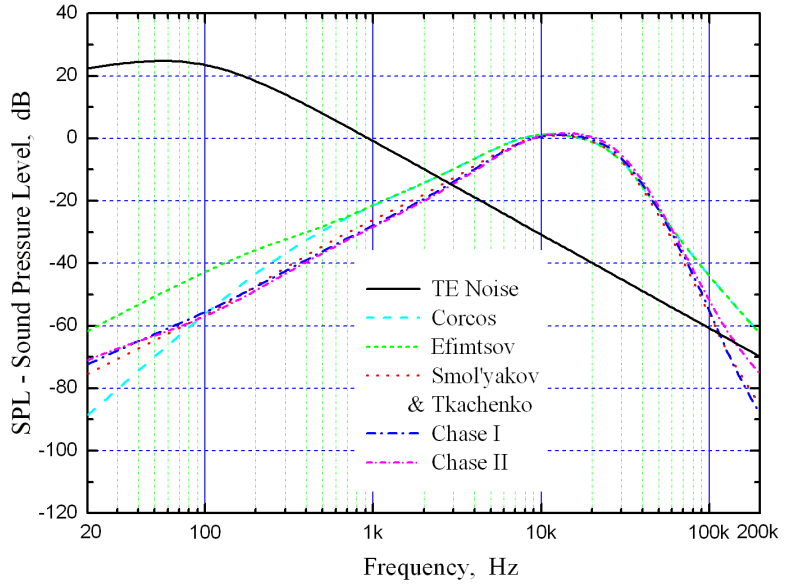

(b) Case (2), $R=0.5 \mathrm{~mm}, \sigma=0.85$

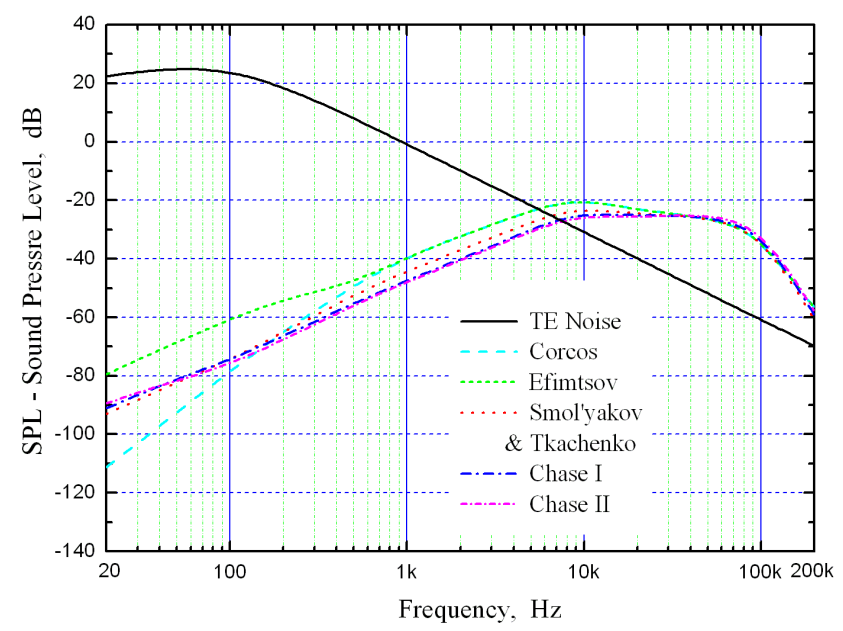

(c) Case (3), $R=0.152 \mathrm{~mm}, \sigma=0.85$

Figure 11. Predicted roughness noise spectra and TE noise spectra for test cases (1)-(3). 
are still remarkably above that of the TE noise spectrum in the frequency range 3-100 kHz. In contrast, Case (3) shows significantly lower roughness noise spectra with the maximum SPL $<-20 \mathrm{~dB}$. This is because the very small roughness elements in Case (3), i.e. $R=0.152 \mathrm{~mm}$, give the dip-galvanized metal surface similar features of a smooth wall. Other possible surface finishes of an aerofoil, e.g., camouflage paint in mass production spraying $(R=0.0305 \mathrm{~mm})$, smooth matt paint $(R=0.0064 \mathrm{~mm}),{ }^{17}$ generate even quieter roughness noise and are therefore not considered further here.

Attention is then confined to the audio frequency range, $f \in[20 \mathrm{~Hz}-20 \mathrm{kHz}]$. Table 2 presents the predicted roughness noise OASPL in this frequency range and it proves the same comparative relationship between the roughness noise and TE noise. For Case (1), the roughness noise OASPL is higher than the TE noise OASPL by $20 \mathrm{dBA}$ in average. Similarly, the predicted OASPL of Case (2) shows a much smaller difference of $3.5 \mathrm{dBA}$ or so between the roughness noise and TE noise. In Case (3), however, the OASPL of roughness noise decreases considerably due to the very small roughness height, and becomes 17.5-23 dBA lower than that of the TE noise. These features tend to support the speculation that the surface roughness generated noise would contribute substantially to the airframe noise of a "clean" configuration provided that the roughness elements are not too small in size.

Table 2. Predicted roughness noise OASPL for test cases (1)-(3). Empirical models for smooth-wall pressure spectrum, 1 - Corcos, 2 - Efimtsov, 3 - Smol'yakov and Tkachenko, 4 - Chase I, 5 - Chase II.

\begin{tabular}{ccrrrrr} 
& \multicolumn{8}{c}{ Roughness Noise } \\
OASPL, dBA & 1 & \multicolumn{1}{c}{2} & \multicolumn{1}{c}{3} & \multicolumn{1}{c}{4} & \multicolumn{1}{c}{5} & TE Noise \\
\hline Case (1) & 49.67 & 49.65 & 49.30 & 50.19 & 51.25 & 30.04 \\
Case (2) & 33.83 & 33.83 & 33.06 & 32.97 & 33.13 & 30.04 \\
Case (3) & 12.62 & 12.62 & 9.53 & 7.78 & 7.04 & 30.04
\end{tabular}

\section{Conclusions}

Howe ${ }^{4}$ has developed a theory of sound generation by turbulent boundary layer flow over a rough wall in which the surface roughness is modeled by a distribution of rigid, hemispherical bosses on a rigid plane. The roughness noise is attributed to the diffraction of the turbulence near field by the bosses, and calculated by means of conventional asymptotic approximation. An empirical model was also proposed by $\mathrm{Howe}^{22}$ by curve-fitting Hersh's experimental data. ${ }^{14}$

In this paper, the diffraction theory has been extended to numerically quantify the radiated roughness noise from a finite surface area to the far field using available empirical models of smooth-wall wavenumberfrequency spectrum scaled by the increased friction velocity and boundary layer thickness for a rough surface. The objective has been to ascertain the reliability of this solution scheme and the possible importance of surface roughness on airframe noise. Acoustic measurements have been performed for the experimental validation. The cross spectra measured by the $1 / 2$ " free-field condenser microphones have shown higher roughness noise than the noise from the smooth plate in $1-2.5 \mathrm{kHz}$ frequency. Also, the source maps through the phased microphone array have indicated that in this frequency range the sound sources on the rough region are at least 8-10 dB stronger than those on the smooth plate. As concluded, comparisons of predicted roughness noise spectra with both the experimental results and the empirical model of Howe ${ }^{22}$ have provided preliminary confirmation of the validity of the diffraction theory and solution scheme.

In the numerical prediction for the far-field radiated roughness noise of a Boeing 757 sized aircraft wing, the initial parametric study has indicated that both the roughness height and roughness density have significant effects on the roughness noise with the former affects the roughness noise more dominantly. The absolute levels of the roughness noise SPL and OASPL for three typical test cases have been approximately quantified. It has been shown that for the three test cases the TE noise is only significant at very low frequencies, whereas the roughness noise turns out to be dominant noise of a "clean" airframe in the high frequency region. Moreover, the roughness noise spectra exceed the TE noise spectra at sufficiently high frequencies, and the OASPL of the former is about $20 \mathrm{dBA}$ and $3.5 \mathrm{dBA}$ higher than the latter for test cases (1) and (2), respectively. Therefore the significant effects of surface roughness on airframe noise need to be carefully considered in the design of a low-noise airframe. 


\section{Appendix: Empirical Models for Wall Pressure Wavenumber-Frequency Spectrum}

The empirical models for the wavenumber-frequency spectrum of turbulent boundary layer pressures on a smooth wall are listed as follows. This is based on the model comparison by Graham, ${ }^{6}$ but only the formulae and constant values are given for quick reference. For the sake of clarity the original notations are accordingly altered to be consistent with the definitions of this paper.

\section{A. The Corcos $\mathrm{Model}^{7}$}

$$
\tilde{\Phi}_{p}(\boldsymbol{\kappa}, \omega)=\frac{4 \alpha_{1} \alpha_{3}}{\left[\alpha_{1}^{2}+\left(U_{c} \kappa_{1} / \omega-1\right)^{2}\right]\left[\alpha_{3}^{2}+U_{c}^{2} \kappa_{3}^{2} / \omega^{2}\right]},
$$

where $\alpha_{1}$ and $\alpha_{3}$ are parameters chosen to yield the best agreement with experiment. Various values for $\alpha_{1}$ and $\alpha_{3}$ are given in the literature; here $\alpha_{1}=0.1$ and $\alpha_{3}=0.77$ are used as suggested by Graham. ${ }^{6}$

\section{B. The Efimtsov Model $^{8}$}

$$
\tilde{\Phi}_{p}(\boldsymbol{\kappa}, \omega)=\frac{\omega^{2}}{U_{c}^{2}} \frac{4 \Lambda_{1} \Lambda_{3}}{\left[1+\Lambda_{1}^{2}\left(\kappa_{1}-\omega / U_{c}\right)^{2}\right]\left[1+\Lambda_{3}^{2} \kappa_{3}^{2}\right]},
$$

where the correlation lengths, $\Lambda_{1}=U_{c} /|\omega| \alpha_{1}$ and $\Lambda_{3}=U_{c} /|\omega| \alpha_{3}$ are given by the empirical expressions

$$
\begin{aligned}
& \frac{\Lambda_{1}}{\delta}=\left[\left(\frac{a_{1} S h}{U_{c} / u_{\tau}}\right)^{2}+\frac{a_{2}^{2}}{S h^{2}+\left(a_{2} / a_{3}\right)^{2}}\right]^{-1 / 2} ; \\
& \frac{\Lambda_{3}}{\delta}=\left\{\begin{array}{c}
{\left[\left(\frac{a_{4} S h}{U_{c} / u_{\tau}}\right)^{2}+\frac{a_{5}^{2}}{S h^{2}+\left(a_{5} / a_{6}\right)^{2}}\right]^{-1 / 2}, \quad M<0.75,} \\
{\left[\left(\frac{a_{4} S h}{U_{c} / u_{\tau}}\right)^{2}+a_{7}^{2}\right]^{-1 / 2}, \quad M>0.9 ;}
\end{array}\right.
\end{aligned}
$$

where $M$ is the free stream Mach number, and $S h=\omega \delta / u_{\tau}$ is the Strouhal number. The constants $a_{1}-a_{7}$ are, respectively,

$$
a_{1}=0.1, \quad a_{2}=72.8, \quad a_{3}=1.54, \quad a_{4}=0.77, \quad a_{5}=548, \quad a_{6}=13.5, \quad a_{7}=5.66 .
$$

Values for $\Lambda_{3}$ when $0.75<M<0.9$ are not given; here interpolation is used if necessary.

\section{The Smol'yakov and Tkachenko Model $^{9}$}

$$
\tilde{\Phi}_{p}(\boldsymbol{\kappa}, \omega)=0.974 A(\omega) h(\omega)[F(\boldsymbol{\kappa}, \omega)-\Delta F(\boldsymbol{\kappa}, \omega)],
$$

where

$$
\begin{aligned}
A(\omega) & =0.124\left[1-\frac{U_{c}}{4 \omega \delta^{*}}+\left(\frac{U_{c}}{4 \omega \delta^{*}}\right)^{2}\right]^{1 / 2}, \\
h(\omega) & =\left[1-\frac{m_{1} A}{6.515 \sqrt{G}}\right]^{-1}, \\
m_{1} & =\frac{1+A^{2}}{1.025+A^{2}}, \quad G=1+A^{2}-1.005 m_{1}, \\
F(\kappa, \omega) & =\left[A^{2}+\left(1-\frac{\kappa_{1} U_{c}}{\omega}\right)^{2}+\left(\frac{\kappa_{3} U_{c}}{6.45 \omega}\right)^{2}\right]^{-3 / 2}, \\
\Delta F(\kappa, \omega) & =0.995\left[1+A^{2}+\frac{1.005}{m_{1}}\left\{\left(m_{1}-\frac{\kappa_{1} U_{c}}{\omega}\right)^{2}+\left(\frac{\kappa_{3} U_{c}}{\omega}\right)^{2}-m_{1}^{2}\right\}\right]^{-3 / 2} .
\end{aligned}
$$

Here $\delta^{*}$ is the boundary layer displacement thickness, taken as $\delta^{*}=\delta / 8$. 


\section{The Ffowcs Williams Model ${ }^{10}$}

$$
\tilde{\Phi}_{p}(\boldsymbol{\kappa}, \omega)=\left(\frac{U_{c}|\boldsymbol{\kappa}|}{\omega}\right)^{2} \frac{4 \alpha_{1} \alpha_{3}}{\left[\alpha_{1}^{2}+\left(U_{c} \kappa_{1} / \omega-1\right)^{2}\right]\left[\alpha_{3}^{2}+U_{c}^{2} \kappa_{3}^{2} / \omega^{2}\right]},
$$

with the same values for $\alpha_{1}$ and $\alpha_{3}$ in the Corcos model.

\section{E. The Chase I Model ${ }^{11}$}

$$
\tilde{\Phi}_{p}(\boldsymbol{\kappa}, \omega)=\frac{\rho_{0}^{2} \omega^{2} u_{\tau}^{3}}{U_{c}^{2} \Phi(\omega)}\left(\frac{C_{M} \kappa_{1}^{2}}{\left[K_{+}^{2}+\left(b_{M} \delta\right)^{-2}\right]^{5 / 2}}+\frac{C_{T}|\boldsymbol{\kappa}|^{2}}{\left[K_{+}^{2}+\left(b_{T} \delta\right)^{-2}\right]^{5 / 2}}\right),
$$

where

$$
\begin{aligned}
K_{+}^{2} & =\frac{\left(\omega-U_{c} \kappa_{1}\right)^{2}}{h^{2} u_{\tau}^{2}}+|\kappa|^{2}, \\
\frac{\Phi(\omega)}{2 \pi} & =\frac{2 \pi h \rho_{0}^{2} u_{\tau}^{4}}{3 \omega\left(1+\mu^{2}\right)}\left(C_{M} F_{M}+C_{T} F_{T}\right), \\
F_{M} & =\frac{1+\mu^{2} \alpha_{M}^{2}+\mu^{4}\left(\alpha_{M}^{2}-1\right)}{\left[\alpha_{M}^{2}+\mu^{2}\left(\alpha_{M}^{2}-1\right)\right]^{3 / 2}}, \\
F_{T} & =\frac{1+\alpha_{T}^{2}+\mu^{2}\left(3 \alpha_{T}^{2}-1\right)+2 \mu^{4}\left(\alpha_{T}^{2}-1\right)}{\left[\alpha_{T}^{2}+\mu^{2}\left(\alpha_{T}^{2}-1\right)\right]^{3 / 2}}, \\
\alpha_{M \text { or } T}^{2} & =1+\left(U_{c} / b_{M \text { or } T} \omega \delta\right)^{2}, \quad \mu=h u_{\tau} / U_{c} .
\end{aligned}
$$

Based on Chase's recommendations, the constants have values as below,

$$
C_{M}=0.0745, \quad C_{T}=0.0475, \quad b_{M}=0.756, \quad b_{T}=0.378, \quad h=3.0 .
$$

\section{F. The Chase II Model ${ }^{12}$}

$$
\tilde{\Phi}_{p}(\boldsymbol{\kappa}, \omega)=\frac{\rho_{0}^{2} \omega^{2} u_{\tau}^{3}}{U_{c}^{2} \Phi(\omega)\left[K_{+}^{2}+(b \delta)^{-2}\right]^{5 / 2}}\left(C_{M} \kappa_{1}^{2}+C_{T}|\boldsymbol{\kappa}|^{2} \frac{K_{+}^{2}+(b \delta)^{-2}}{|\boldsymbol{\kappa}|^{2}+(b \delta)^{-2}}\right),
$$

where

$$
\begin{aligned}
K_{+}^{2} & =\frac{\left(\omega-U_{c} \kappa_{1}\right)^{2}}{h^{2} u_{\tau}^{2}}+|\boldsymbol{\kappa}|^{2}, \\
\frac{\Phi(\omega)}{2 \pi} & =\frac{2 \pi h \rho_{0}^{2} u_{\tau}^{4}}{3 \omega\left(1+\mu^{2}\right)}\left(C_{M} F_{M}+C_{T} F_{T}\right), \\
F_{M} & =\frac{1+\mu^{2} \alpha^{2}+\mu^{4}\left(\alpha^{2}-1\right)}{\left[\alpha^{2}+\mu^{2}\left(\alpha^{2}-1\right)\right]^{3 / 2}}, \\
F_{T} & =\frac{3\left(1+\mu^{2}\right)\left(1+\alpha^{2}\right)}{2 \alpha^{3}}, \\
\alpha^{2} & =1+\left(U_{c} / b \omega \delta\right)^{2}, \quad \mu=h u_{\tau} / U_{c} .
\end{aligned}
$$

The recommended constant values are,

$$
b=0.75, \quad h=3.0, \quad h C_{M}=0.466, \quad h C_{T}=0.014 .
$$

\section{Acknowledgments}

This work is part of a collaborative project, Silent Aircraft Initiative (SAI), funded by the CambridgeMIT Institute (CMI) whose support is gratefully acknowledged. The author would also like to thank the Overseas Research Studentship (ORS) and the Gates Cambridge Scholarship which have made this research possible. Moreover, special thanks go to Dr. Will Graham in Department of Engineering for helpful advice on the form of the smooth-wall pressure spectrum. 


\section{References}

${ }^{1}$ Faszer, A. and Hileman, J., "Modelling Minimum Airframe Noise for an All-Lifting-Body," 8th ASC-CEAS Workshop, Budapest, November 2004.

${ }^{2}$ Franzoni, L., "Assessment of the Contribution of Panel Vibration to Airframe Noise," AIAA Journal, Vol. 42, No. 8, August 2004, pp. 1522-1528.

${ }^{3}$ Schlichting, H., Boundary Layer Theory, McGraw-Hill, New York, seventh ed., 1979.

${ }^{4}$ Howe, M. S., "On the Generation of Sound by Turbulent Boundary Layer Flow over a Rough Wall," Proceedings of the Royal Society of London, Vol. A395, 1984, pp. 247-263.

${ }^{5}$ Howe, M. S., "The Influence of Viscous Surface Stress on the Production of Sound by a Turbulent Boundary Layer over a Rough Wall," Journal of Sound and Vibration, Vol. 104, No. 1, 1986, pp. 29-39.

${ }^{6}$ Graham, W. R., "A Comparison of Models for the Wavenumber-Frequency Spectrum of Turbulent Boundary Layer Pressures," Journal of Sound and Vibration, Vol. 206, No. 4, 1997, pp. 541-565.

${ }^{7}$ Corcos, G. M., "The Structure of the Turbulent Pressure Field in Boundary- Layer Flows," Journal of Fluid Mechanics, Vol. 18, No. 3, 1964, pp. 353-378.

${ }^{8}$ Efimtsov, B. M., "Characteristics of the Field of Turbulent Wall Pressure Fluctuations at Large Reynolds Numbers," Soviet Physics Acoustics, Vol. 28, No. 4, 1982, pp. 289-292.

${ }^{9}$ Smol'yakov, A. V. and Tkachenko, V. M., "Model of a Field of Pseudosonic Turbulent Wall Pressures and Experimental Data," Soviet Physics Acoustics, Vol. 37, No. 6, 1991, pp. 627-631.

${ }^{10}$ Williams, J. E. F., "Boundary-Layer Pressures and the Corcos Model: a Development to Incorporate Low Wavenumber Constraints," Journal of Fluid Mechanics, Vol. 125, 1982, pp. 9-25.

${ }^{11}$ Chase, D. M., "Modeling the Wavevector-Frequency Spectrum of Turbulent Boundary Layer Wall Pressure," Journal of Sound and Vibration, Vol. 70, No. 1, 1980, pp. 29-67.

${ }^{12}$ Chase, D. M., "The Character of the Turbulent Wall Pressure Spectrum at Subconvective Wavenumbers and a Suggested Comprehensive Model," Journal of Sound and Vibration, Vol. 112, No. 1, 1987, pp. 125-147.

${ }^{13}$ Howe, M. S., "The Turbulent Boundary-Layer Rough-Wall Pressure Spectrum at Acoustic and Subconvective Wavenumbers," Proceedings of the Royal Society of London, Vol. A415, 1988, pp. 141-161.

${ }^{14}$ Hersh, A. S., "Surface Roughness Generated Flow Noise," AIAA Paper 83-0786, 1983.

${ }^{15}$ Crighton, D. G., Dowling, A. P., Williams, J. E. F., Heckl, M., and Leppington, F. G., Modern Methods in Analytical Acoustics: Lecture Notes, Springer-Verlag, London, 1992.

${ }^{16}$ Blake, W. K., Mechanics of Flow-Induced Sound and Vibration, Vol. II: Complex Flow-Structure Interactions, Academic Press, New York, 1986.

17 "The Mean Skin Friction Coefficient for a Rough Flat Plate with a Turbulent Two-Demensional Boundary Layer in Compressible Adiabatic Flow, with Application to Wedges, Cylinders and Cones," ESDU Aerodynamics Data Item 2c-73016, 1973.

${ }^{18}$ Prandtl, L. and Schlichting, H., Werft-Reederei-Hafen, Part I, 1934.

${ }^{19}$ Nikuradse, J., Forsch.-Arb. Ing.-Wesen, Heft 361, 1933.

${ }^{20}$ Mills, A. F. and Hang, X., "On the Skin Friction Coefficient for a Fully Rough Flat Plate," ASME Journal of Fluids Engineering, Vol. 105, pp. 364-365.

${ }^{21}$ Krogstad, P. A., Antonia, R. A., and Browne, L. W. B., "Comparison between Rough- and Smooth-Wall Turbulent Boundary Layers," Journal of Fluid Mechanics, Vol. 245, 1992, pp. 599-617.

${ }^{22}$ Howe, M. S., Acoustics of Fluid-Structure Interactions, Cambridge University Press, Cambridge, 1998.

${ }^{23}$ Ahn, B., Modeling Unsteady Wall Pressures beneath Turbulent Boundary Layers, Ph.D. thesis, Department of Engineering, University of Cambridge, 2005.

${ }^{24}$ Blake, W. K., "Turbulent Bounday-Layer Wall-Pressure Fluctuations on Smooth and Rough Walls," Journal of Fluid Mechanics, Vol. 44, 1970, pp. 637-660.

${ }^{25}$ Duncan, W. J., Thom, A. S., and Young, A. D., Mechanics of Fluids, Edward Arnold Ltd., London, 2nd ed., 1970.

${ }^{26}$ Dowling, A. P. and Williams, J. E. F., Sound and Sources of Sound, Ellis Horwood Ltd., Chichester, 1983. 